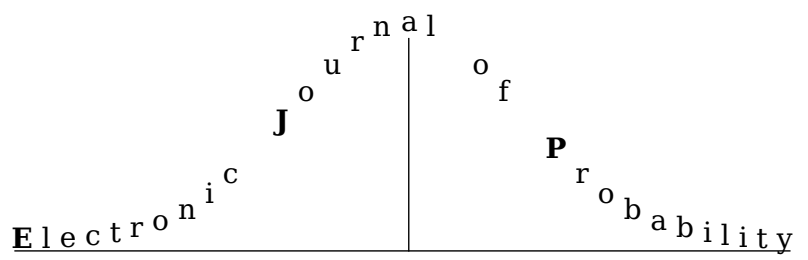

Electron. J. Probab. 26 (2021), article no. 140, 1-37.

ISSN: 1083-6489 https://doi.org/10.1214/21-EJP690

\title{
Spatial ergodicity for SPDEs via Poincaré-type inequalities*
}

\author{
Le Chen ${ }^{\dagger} \quad$ Davar Khoshnevisan ${ }^{\ddagger} \quad$ David Nualart ${ }^{\S} \quad$ Fei Pu
}

\begin{abstract}
Consider a parabolic stochastic PDE of the form $\partial_{t} u=\frac{1}{2} \Delta u+\sigma(u) \eta$, where $u=u(t, x)$ for $t \geq 0$ and $x \in \mathbb{R}^{d}, \sigma: \mathbb{R} \rightarrow \mathbb{R}$ is Lipschitz continuous and non random, and $\eta$ is a centered Gaussian noise that is white in time and colored in space, with a possiblysigned homogeneous spatial correlation $f$. If, in addition, $u(0) \equiv 1$, then we prove that, under a mild decay condition on $f$, the process $x \mapsto u(t, x)$ is stationary and ergodic at all times $t>0$. It has been argued that, when coupled with moment estimates, spatial ergodicity of $u$ teaches us about the intermittent nature of the solution to such SPDEs [1, 37]. Our results provide rigorous justification of such discussions.

Our methods hinge on novel facts from harmonic analysis and functions of positive type, as well as from Malliavin calculus and Poincaré inequalities. We further showcase the utility of these Poincaré inequalities by: (a) describing conditions that ensure that the random field $u(t)$ is mixing for every $t>0$; and by (b) giving a quick proof of a conjecture of Conus et al [15] about the "size" of the intermittency islands of $u$.

The ergodicity and the mixing results of this paper are sharp, as they include the classical theory of Maruyama [42] (see also Dym and McKean [23]) in the simple setting where the nonlinear term $\sigma$ is a constant function.
\end{abstract}

Keywords: SPDEs; ergodicity; Malliavin calculus; Poincaré-type inequality. MSC2020 subject classifications: $60 \mathrm{H} 15 ; 37 \mathrm{~A} 25 ; 60 \mathrm{H} 07 ; 60 \mathrm{G} 10$.

Submitted to EJP on February 27, 2021, final version accepted on August 23, 2021.

\section{Introduction}

The principal aim of this article is to establish relatively simple-to-check, but also broad, conditions under which the solution $u=\{u(t, x)\}_{t>0, x \in \mathbb{R}^{d}}$ to a parabolic stochastic PDE is spatially stationary and ergodic. Equivalently, we would like to know conditions under which $u(t)$ is stationary and ergodic, in its spatial variable $x$, at all times $t>0$. This

\footnotetext{
${ }^{*}$ Research supported in part by NSF grants DMS-1811181 (D.N.) and DMS-1855439 (D.K.)

${ }^{\dagger}$ Emory University, United States of America. E-mail: le. chen@emory.edu

¥University of Utah, United States of America. E-mail: davar@math.utah.edu

${ }^{\S}$ University of Kansas, United States of America. E-mail: nualart@ku.edu

"Beijing Normal University, China. E-mail: pufeibnu@gmail .com
} 
problem, and its relation to intermittency, have been mentioned informally for example in the introduction of Bertini and Cancrini [1] (see also [37, Ch. 7]). This problem is also connected somewhat loosely to novel applications of Malliavin calculus to central limit theorems for parabolic SPDEs; see Huang et al [33, 34].

In order for spatial ergodicity to be a meaningful property, one needs to consider parabolic SPDEs for which the solution is a priori a stationary process in its spatial variable. Thus, we study the following archetypal parabolic problem:

$$
\left\{\begin{array}{l}
\partial_{t} u=\frac{1}{2} \Delta u+\sigma(u) \eta \quad \text { on }(0, \infty) \times \mathbb{R}^{d} \\
u(0) \equiv 1
\end{array}\right.
$$

where $\sigma \not \equiv 0$ is Lipschitz continuous and non random, and $\eta$ denotes a generalized, centered, Gaussian random field with covariance form

$$
\mathrm{E}[\eta(t, x) \eta(s, y)]=\delta_{0}(t-s) f(x-y) \quad \text { for all } s, t \geq 0 \text { and } x, y \in \mathbb{R}^{d},
$$

and where $f$ is a nonnegative-definite distribution on $\mathbb{R}^{d}$. Somewhat more formally, the Wiener-integral process $\psi \mapsto \eta(\psi):=\int_{\mathbb{R}_{+} \times \mathbb{R}^{d}} \psi(t, x) \eta(\mathrm{d} t \mathrm{~d} x)$ is linear a.s. and satisfies

$$
\operatorname{Cov}\left(\eta\left(\psi_{1}\right), \eta\left(\psi_{2}\right)\right)=\int_{0}^{\infty}\left\langle\psi_{1}(t), \psi_{2}(t) * f\right\rangle_{L^{2}\left(\mathbb{R}^{d}\right)} \mathrm{d} t,
$$

for every $\psi_{1}, \psi_{2}$ in the space $C_{c}\left(\mathbb{R}_{+} \times \mathbb{R}^{d}\right)$ of all compactly-supported, continuous, realvalued functions on $\mathbb{R}_{+} \times \mathbb{R}^{d}$.

The solution theory for (1.1) is particularly well established when the spatial correlation $f$ of the noise $\eta$ belongs to the space $\mathfrak{M}_{+}\left(\mathbb{R}^{d}\right)$ of all nonnegative-definite tempered Borel measures on $\mathbb{R}^{d}$. In that case, it is well known that the Fourier transform is a one-to-one linear mapping from $\mathfrak{M}_{+}\left(\mathbb{R}^{d}\right)$ to itself. That is, $f \in \mathfrak{M}_{+}\left(\mathbb{R}^{d}\right)$ if and only if $\hat{f} \in \mathfrak{M}_{+}\left(\mathbb{R}^{d}\right){ }^{1}$ and

$$
\int_{\mathbb{R}^{d}} \phi \mathrm{d} f=\int_{\mathbb{R}^{d}} \overline{\hat{\phi}} \mathrm{d} \hat{f}=\int_{\mathbb{R}^{d}} \hat{\phi} \mathrm{d} \hat{f} \quad \text { for all } \phi \in \mathscr{S}\left(\mathbb{R}^{d}\right),
$$

where $\mathscr{S}\left(\mathbb{R}^{d}\right)$ denotes the usual space of all test functions of rapid decrease on $\mathbb{R}^{d}$. The measure $\hat{f}$ is sometimes called the spectral measure of $f$. And the theory of Dalang [19] implies that if

$$
f \in \mathfrak{M}_{+}\left(\mathbb{R}^{d}\right), \quad \text { and } \quad \int_{\mathbb{R}^{d}} \frac{\hat{f}(\mathrm{~d} x)}{\lambda+\|x\|^{2}}<\infty \text { for one }- \text { hence all }-\lambda>0,
$$

then (1.1) has a random-field solution $u$ that is unique subject to the following integrability condition:

$$
\sup _{t \in[0, T]} \sup _{x \in \mathbb{R}^{d}} \mathrm{E}\left(|u(t, x)|^{k}\right)<\infty \quad \text { for every } T>0 \text { and } k \geq 2 .
$$

Moreover, $(t, x) \mapsto u(t, x)$ is $L^{k}(\Omega)$-continuous. Furthermore, it is known that Condition (1.4) is necessary and sufficient for example when $\sigma$ is a non-zero constant; see Dalang [19], as well as Peszat and Zabczyk [50].

Let

$$
\mathbb{B}_{r}:=\left\{x \in \mathbb{R}^{d}:\|x\| \leq r\right\} \quad \text { for every } r>0 .
$$

Our first result is a detailed description of the spatial ergodicity of $u$ in the case that $f$ satisfies Dalang's condition (1.4).

\footnotetext{
${ }^{1}$ the Fourier transform so that $\hat{\psi}(x)=\int_{\mathbb{R}^{d}} \exp (i x \cdot y) \psi(y) \mathrm{d} y$ for all $\psi \in L^{1}\left(\mathbb{R}^{d}\right)$ and $x \in \mathbb{R}^{d}$.
} 
Theorem 1.1. If $f$ satisfies (1.4), then $u(t)=\{u(t, x)\}_{x \in \mathbb{R}^{d}}$ is a stationary random field for every $t>0$. Moreover, the following are valid:

1. If $\hat{f}(\{0\})=0$, then $u$ is spatially ergodic;

2. $\hat{f}(\{0\})=0$ iff $f\left(\mathbb{B}_{r}\right)=o\left(r^{d}\right)$ as $r \rightarrow \infty$;

3. If $\sigma$ is a nonzero constant, then $\hat{f}(\{0\})=0$ iff $u$ is spatially ergodic;

4. $\hat{f}(\{0\})>0$ iff $\hat{f}$ has an atom.

If $f$ is a function that satisfies (1.4), then part 2 of Theorem 1.1 can be recast as follows:

$$
\hat{f}(\{0\})=0 \text { if and only if } \lim _{r \rightarrow \infty} \frac{1}{\left|\mathbb{B}_{r}\right|} \int_{\mathbb{B}_{r}} f(x) \mathrm{d} x=0 .
$$

Thus, we see that when $f$ is a function, $\hat{f}(\{0\})=0$ iff the asymptotic average of $f$ is zero.

Remark 1.2. Maruyama [42] has shown that if a 1-parameter, stationary Gaussian process $X=\left\{X_{t}\right\}_{t \in \mathbb{R}}$ has a continuous covariance function $\varrho$, then $X$ is ergodic if and only if $\hat{\varrho}$ has no atoms; see also Dym and McKean [23, §3.9]. When $d=1$, Part 3 of Theorem 1.1 can be derived easily by verifying Maruyama's condition, using part 4 of Theorem 1.1; part 4 of Theorem 1.1 and its connection to mean-zero property (1.7) of $f$ appear to be new, at this level of generality, even in the Gaussian case.

There is also a literature on well-posedness and regularity theory for (1.1) when $f$ is a distribution that is not necessarily in $\mathfrak{M}_{+}\left(\mathbb{R}^{d}\right)$, though such results tend to be applicable in a more specialized setting as compared with the theory of Dalang [19]; see for example $[13,11,30,31,32]$. Henceforth, we consider the case that $f$ is a nonnegative-definite, but possibly signed, function of the form,

$$
f=h * \tilde{h},
$$

where $h: \mathbb{R}^{d} \rightarrow \mathbb{R}$ has enough regularity to ensure among other things that the convolution in (1.8) is well defined, and $\tilde{h}(x):=h(-x)$ defines the reflection of $h$. In this case, (1.2) is equivalent to the elegant formula

$$
\operatorname{Var}(\eta(\psi))=\int_{0}^{\infty}\|\psi(t) * h\|_{L^{2}\left(\mathbb{R}^{d}\right)}^{2} \mathrm{~d} t, \quad \text { valid for all } \psi \in C_{c}\left(\mathbb{R}_{+} \times \mathbb{R}^{d}\right) .
$$

In this context, we prove that a mild integrability condition on $h$ implies that $|h| \epsilon$ $H_{-1}\left(\mathbb{R}^{d}\right)$ - see (1.10) and Lemma 3.5 - which in turn implies the existence of a spatially stationary random-field solution $u$ to (1.1) that is unique subject to (1.5); see Theorem 5.3. More significantly, we prove that the ensuing Condition (1.10) on $h$ ensures that $u$ is spatially ergodic.

In any case, the end result is the following theorem. ${ }^{2}$ In order to present that result we first recall (1.6), and then define for every $r>0$,

$$
\omega_{d}(r):= \begin{cases}1 & \text { if } d=1, \\ r \log _{+}(1 / r) & \text { if } d=2, \\ r & \text { if } d \geq 3,\end{cases}
$$

where $\log _{+}(z):=\log (z \vee \mathrm{e})$ for all $z \in \mathbb{R}$.

Theorem 1.3. Assume that the spatial correlation function $f: \mathbb{R}^{d} \rightarrow \mathbb{R}$ satisfies (1.8), where $h \in L_{\text {loc }}^{p}\left(\mathbb{R}^{d}\right)$ for some number $p>1$, and satisfies

$$
\int_{0}^{1}\left(\|h\|_{L^{p}\left(\mathbb{B}_{r}\right)}\|h\|_{L^{q}\left(\mathbb{B}_{r}^{c}\right)}+\|h\|_{L^{2}\left(\mathbb{B}_{r}^{c}\right)}^{2}\right) \omega_{d}(r) \mathrm{d} r<\infty \quad \text { with } \quad q:=\frac{p}{p-1} .
$$

\footnotetext{
${ }^{2}$ For a very brief discussion of relevant measurability issues, see Remark 5.5 below.
} 
Then the SPDE (1.1) has a spatially stationary and ergodic random-field solution $u$ that is unique subject to the integrability condition (1.5).

Remark 1.4. In order to be concrete, we have selected the initial data to be identically one in Theorems 1.1 and 1.3. The same arguments show that Theorems 1.1 and 1.3 continue to hold when the initial data is an arbitrary stationary random field $\{u(0, x)\}_{x \in \mathbb{R}^{d}}$ that is independent of $\eta$ and is continuous in $L^{k}(\Omega)$ for every real number $k \geq 1$.

In the case $f$ is signed and satisfies (1.8), the following presents easy-to-check conditions on $h$ in order for (1.1) to have a unique random-field solution that is spatially ergodic (as well as stationary).

Corollary 1.5. Suppose that $h: \mathbb{R}^{d} \rightarrow \mathbb{R}$ is Borel measurable, and either that $h \in L^{2}\left(\mathbb{R}^{d}\right)$ or that there exist $\alpha \in(0, d \wedge 2)$ and $\beta>0$ such that

$$
\sup _{\|w\|<1}\|w\|^{(d+\alpha) / 2}|h(w)|<\infty \text { and } \sup _{\|z\|>1}\|z\|^{(d+\beta) / 2}|h(z)|<\infty .
$$

Then (1.1) has a random-field solution $u$ that is unique subject to the moment condition (1.5). Moreover, $u(t)$ is stationary and ergodic for every $t>0$.

It is worth noting that, whereas (1.10) is a global integrability condition on $h,(1.11)$ involves: (i) A local condition on the behavior of $h$ near the origin; and (ii) A separate localat-infinity (growth) condition on $h$. We will show quickly in $\S 8$ that (1.11) implies (1.10).

It is also worth noting that the first (local) condition on $h$ in (1.11) is there merely to ensure that $|h| \in H_{-1}\left(\mathbb{R}^{d}\right)$, which in turn will imply that (1.1) has a solution. The second (growth) condition on $h$ in (1.11) is the more interesting hypothesis. That condition is responsible for ensuring that $h$ - whence also $f$ - decays sufficiently rapidly so that spatial ergodicity of the solution $u$ to (1.1) is ensured.

Our ergodicity results (Theorems 1.1 and 1.3) are consequences of the following two Poincaré-type inequalities.

Theorem 1.6 (Poincaré inequality I). Assume that the conditions of Theorem 1.1 are met. Then for every number $T>0$ there exists a real number $C>0$ such that

$$
\sup _{t \in[0, T]} \operatorname{Var}\left(\frac{1}{N^{d}} \int_{[0, N]^{d}} \prod_{j=1}^{k} g_{j}\left(u\left(t, x+\zeta^{j}\right)\right) \mathrm{d} x\right) \leq \frac{C k^{2}}{N^{d}} f\left([-N, N]^{d}\right),
$$

uniformly for every integer $k \geq 1$, real number $N>1, \zeta^{1}, \ldots, \zeta^{k} \in \mathbb{R}^{d}$, and all Lipschitzcontinuous functions $g_{1}, \ldots, g_{k}: \mathbb{R} \rightarrow \mathbb{R}$ that satisfy

$$
g_{j}(0)=0 \quad \text { and } \quad \operatorname{Lip}\left(g_{j}\right)=1 \quad \text { for every } j=1, \ldots, k .
$$

Here we are using the standard convention that $\operatorname{Lip}(\psi)$ denotes the Lipschitz constant of $\psi: \mathbb{R}^{d} \rightarrow \mathbb{R}$; that is,

$$
\operatorname{Lip}(\psi):=\sup _{x \neq y} \frac{|\psi(x)-\psi(y)|}{\|x-y\|} .
$$

Theorem 1.7 (Poincaré inequality II). Assume that the conditions of Theorem 1.3 are met. Then for every number $T>0$ there exists a real number $C>0$ such that

$$
\sup _{t \in[0, T]} \operatorname{Var}\left(\frac{1}{N^{d}} \int_{[0, N]^{d}} \prod_{j=1}^{k} g_{j}\left(u\left(t, x+\zeta^{j}\right)\right) \mathrm{d} x\right) \leq \frac{C k^{2}}{N^{d}} \int_{[-N, N]^{d}}(|h| *|\tilde{h}|)(x) \mathrm{d} x,
$$

uniformly for every integer $k \geq 1$, real number $N>1, \zeta^{1}, \ldots, \zeta^{k} \in \mathbb{R}^{d}$, and all Lipschitzcontinuous functions $g_{1}, \ldots, g_{k}: \mathbb{R} \rightarrow \mathbb{R}$ that satisfy (1.13). 
Theorems 1.6 and 1.7 are proved in §8. The proofs make a novel appeal to the Malliavin calculus, specifically to the Clark-Ocone formula; see §6. Next, we would like to explain briefly why Theorems 1.6 and 1.7 are indeed Poincaré-type inequalities, as is suggested also by the title of the paper.

Let $F$ denote a square-integrable random variable in a nice filtered probability space that is rich enough to carry a sufficiently-nice Gaussian measure. In this context, the Poincaré inequality states roughly that one can estimate the variance of $F$ by finding good estimates for the Malliavin derivative of $F$. Capitaine, Hsu, and Ledoux [5] have observed that the Poincaré inequality can be deduced from the Clark-Ocone formula. The argument is elegant and brief. We describe it next in the context of 1-D Brownian motion $B$. Let us construct $B=\left\{B_{t}\right\}_{t \geq 0}$ on the space $\Omega:=C\left(\mathbb{R}_{+} ; \mathbb{R}\right)$ via the coordinate $\operatorname{map}\left[B_{t}(\omega):=\omega(t)\right.$ for all $\omega \in \Omega$ and $\left.t \geq 0\right]$ and Wiener measure W. Let $\left\{\mathcal{B}_{t}\right\}_{t \geq 0}$ denote the natural filtration of $B$, augmented in the usual way. According to the Clark-Ocone formula [14] - see also [46, Proposition 1.3.14]) - if $F \in L^{2}\left(\Omega, \mathcal{B}_{T}, \mathbb{W}\right)$ for some $T>0$, and is in a suitable Gaussian Sobolev space, then $F-\mathrm{E}_{\mathrm{W}} F=\int_{0}^{T} \mathrm{E}_{\mathrm{w}}\left(D_{t} F \mid \mathcal{B}_{t}\right) \mathrm{d} B_{t}$ a.s. [W], where $D F$ denotes the Malliavin derivative of $F$. The Itô isometry, Jensen's inequality, and two back-to-back appeals to Fubini's theorem, together imply that

$$
\operatorname{Var}_{\mathrm{W}}(F)=\mathrm{E}_{\mathrm{W}}\left(\int_{0}^{T}\left|\mathrm{E}_{\mathrm{W}}\left(D_{t} F \mid \mathcal{B}_{t}\right)\right|^{2} \mathrm{~d} t\right) \leq \int_{0}^{T} \mathrm{E}_{\mathrm{W}}\left(\left|D_{t} F\right|^{2}\right) \mathrm{d} t=\mathrm{E}_{\mathrm{W}}\left(\|D F\|_{L^{2}[0, T]}^{2}\right),
$$

which is precisely the classical Poincaré inequality on the Wiener space $\left(\Omega, \vee_{t \geq 0} \mathcal{B}_{t}, \mathbb{W}\right)$. This is one way to state more carefully our earlier assertion that good information on the magnitude of the second moment of $\|D F\|_{L^{2}[0, T]}$ can imply an upper bound on the variance of $F$. Theorems 1.6 and 1.7 are certain specializations of a more complex form of this Poincaré inequality (see Proposition 6.3), wherein the above Brownian motion $B$ is replaced by an infinite dimensional Brownian motion. Theorems 1.6 and 1.7 include also sharp Malliavin derivative bounds, whose derivation requires additional ideas and extra effort.

Here is a brief outline of the paper: In $\$ 2$ we present an example which shows that we cannot expect spatial ergodicity of the solution of (1.1) unless $f$ exhibits some sort of decay at infinity, valid even when $\sigma$ is not constant. Section 3.1 includes comments and a few harmonic-analytic results on functions of positive type. Section 3.2 discusses known results on the well-posedness of (1.1), and discusses how the conditions of Theorem 1.3 ensure among other things that the absolute value of $h$ is in the classical space Hilbert space $H_{-1}\left(\mathbb{R}^{d}\right)$. Section 3.4 contains a quick proof of the folklore fact which is part 4 of Theorem 1.1. In $\$ 5.1$ we extend the stochastic Young inequality of Walsh integrals $[16,25]$ to the case that $f$ is possibly signed and satisfies the conditions of Theorem 1.3. It is shown in $§ 5.2$ that the well-posedness of (1.1) is a ready consequence of the mentioned stochastic Young's inequality; see Theorem 5.3. Methods of Malliavin calculus turn out to play a central role in the study of the spatial ergodicity of the solution, and we present the elements of Malliavin calculus in $§ 6$. The stationarity assertion of Theorem 1.3 is proved next in $\S 7$. Theorem 1.3 is proved shortly following the proof of Theorem 1.6, and in a final section 9, we use our Poincaré inequalities to establish tight criteria for spatial mixing of the solution to (1.1) [§9.1] and also prove a conjecture of Conus et al [15] related to an "intermittency" property of the solution to (1.1) in a special, though important, case [\$9.2].

Let us close the Introduction with a brief description of the notation of this paper. Throughout we write " $g_{1}(x) \lesssim g_{2}(x)$ for all $x \in X$ " when there exists a real number $L$ such that $g_{1}(x) \leq L g_{2}(x)$ for all $x \in X$. Alternatively, we might write " $g_{2}(x) \gtrsim g_{1}(x)$ for all $x \in X$." By " $g_{1}(x) \asymp g_{2}(x)$ for all $x \in X$ " we mean that $g_{1}(x) \lesssim g_{2}(x)$ for all $x \in X$ and 
$g_{2}(x) \lesssim g_{1}(x)$ for all $x \in X$. Finally, " $g_{1}(x) \propto g_{2}(x)$ for all $x \in X$ " means that there exists a real number $L$ such that $g_{1}(x)=L g_{2}(x)$ for all $x \in X$.

Throughout, we write

$$
f_{E} \psi(x) \mathrm{d} x:=\frac{1}{|E|} \int_{E} \psi(x) \mathrm{d} x,
$$

whenever $\psi: \mathbb{R}^{d} \rightarrow \mathbb{R}$ is integrable on a Lebesgue-measurable set $E \subset \mathbb{R}^{d}$ whose Lebesgue measure $|E|$ is strictly positive. We will use the notation, $\|X\|_{k}:=\left\{\mathrm{E}\left(|X|^{k}\right)\right\}^{1 / k}$, valid for every real-valued random variable $X \in L^{k}(\Omega)$ and every real number $k \geq 1$.

\section{A non-ergodic example}

In the Introduction we alluded that if the tails of the spatial correlation $f$ do not vanish, then we cannot generally expect $u(t)$ to be ergodic for all $t \geq 0$. We now describe this in the context of an example in which the spatial correlation function $f(x)$ does not decay as $\|x\| \rightarrow \infty$, the solution $u$ exists and is non-degenerate, and $u$ is not spatially ergodic at positive times.

First, we might as well rule out trivialities by assuming that

$$
\sigma(1) \neq 0 .
$$

Otherwise, one can see easily that $u(t, x) \equiv 1$; in this case, $u(t)$ is ergodic for all $t \geq 0$, but only in a vacuous sense.

Next, let us choose and fix a number $\lambda>0$, and suppose that

$$
f(x)=\lambda^{2} \quad \text { for all } x \in \mathbb{R}^{d},
$$

to ensure that the tails of $f$ do not decay. In this case, it is possible to realize the noise $\eta(\mathrm{d} t \mathrm{~d} x)$ as $\lambda \mathrm{d} W_{t} \mathrm{~d} x$, where $W$ denotes a one-dimensional Brownian motion. Thus, we can infer from (1.1) and well-known arguments that, under (2.2),

$$
u(t, x)=X_{t} \quad \text { for all } t \geq 0 \text { and } x \in \mathbb{R}^{d} \text { a.s. },
$$

where $X$ is the unique (strong) solution of the one-dimensional Itô SDE,

$$
\mathrm{d} X_{t}=\lambda \sigma\left(X_{t}\right) \mathrm{d} W_{t}, \quad \text { subject to } X_{0}=1 .
$$

Standard estimates now reveal that

$$
\lim _{t \rightarrow 0^{+}} \frac{1}{t} \operatorname{Var}\left(X_{t}\right)=\lambda^{2} \sigma^{2}(1),
$$

whence $\operatorname{Var}\left(X_{t}\right)>0$ for all $t$ small. Thus, we conclude from (2.3) that, under conditions (2.1) and (2.2), the process $u$ is not spatially ergodic. In fact, a little more effort shows that $\operatorname{Var}\left(X_{t}\right)>0$ for all $t>0$, thanks to the Markov property. And this implies that $u(t)$ is not ergodic for any $t>0$.

\section{Harmonic analysis}

\subsection{Functions of positive type}

Let us recall the notation from (1.6) for closed centered balls, and recall the following from classical harmonic analysis [35]:

Definition 3.1. We say that a function $g: \mathbb{R}^{d} \rightarrow \mathbb{R}$ is of positive type if:

1. $g$ is locally integrable and nonnegative definite in the sense of distributions (that is, $\hat{g} \geq 0$ and hence a Borel measure, thanks to the Riesz representation theorem); 
2. The restriction of $g$ to $\mathbb{B}_{r}^{c}$ is a uniformly continuous function for every $r>0$; and

3. $\lim _{\|x\| \rightarrow \infty} g(x)=0 .^{3}$

Typical examples include $g(x)=\exp \left(-\alpha\|x\|^{\beta}\right)$ and $g(x)=\left(\alpha^{\prime}+\|x\|^{\beta}\right)^{-1}$, for constants $\alpha \geq 0, \alpha^{\prime}>0$, and $\beta \in(0,2]$. There are also unbounded examples such as Riesz kernels $\left(g(x)=\|x\|^{-\gamma}\right.$ for $\gamma \in(0, d)$ ), as well as products of the preceding such as $g(x)=\|x\|^{-\gamma} \exp \left(-\alpha\|x\|^{\beta}\right)$.

The main goal of this section is to present a family $\cup_{p>1} \mathcal{F}_{p}\left(\mathbb{R}^{d}\right)$ of real-valued functions on $\mathbb{R}^{d}$ that can be used explicitly to construct a large number of functions of positive type that are central to our analysis. We will also use this opportunity to introduce another vector space $\cup_{p>1} \mathcal{G}_{p}\left(\mathbb{R}^{d}\right)$ of functions that will play a prominent role in later sections (though not in this one).

Definition 3.2. Choose and fix a real number $p>1$, and define $\mathcal{F}_{p}\left(\mathbb{R}^{d}\right)$ to be the collection of all $h \in L_{\text {loc }}^{p}\left(\mathbb{R}^{d}\right)$ that satisfy

$$
\int_{0}^{1} s^{d-1}\left(\|h\|_{L^{p}\left(\mathbb{B}_{s}\right)}\|h\|_{L^{q}\left(\mathbb{B}_{s}^{c}\right)}+\|h\|_{L^{2}\left(\mathbb{B}_{s}^{c}\right)}^{2}\right) \mathrm{d} s<\infty \quad \text { with } \quad q:=\frac{p}{p-1} .
$$

We also define $\mathcal{G}_{p}\left(\mathbb{R}^{d}\right)$ to be the collection of all functions $h \in L_{\text {loc }}^{p}\left(\mathbb{R}^{d}\right)$ that satisfy (1.10).

In this section we study some of the basic properties of the elements of the spaces $\cup_{p>1} \mathcal{F}_{p}\left(\mathbb{R}^{d}\right)$ and $\cup_{p>1} \mathcal{G}_{p}\left(\mathbb{R}^{d}\right)$. It might help to add that, notationally speaking, the functions $h$ in $\cup_{p>1} \mathcal{G}_{p}\left(\mathbb{R}^{d}\right)$ and $\cup_{p>1} \mathcal{F}_{p}\left(\mathbb{R}^{d}\right)$ will be potential candidates for the function $h$ in (1.8), which are then used to form the spatial correlation function $f$ in (1.1). Thus, the notation should aid the reading, and not hinder it.

Lemma 3.3. The following are valid for every $p>1$, where $q:=p /(p-1)$ :

1. $\mathcal{G}_{p}\left(\mathbb{R}^{d}\right) \subseteq \mathcal{F}_{p}\left(\mathbb{R}^{d}\right) \subseteq L_{\text {loc }}^{1}\left(\mathbb{R}^{d}\right)$ for all $d \geq 1$, and $\mathcal{G}_{p}(\mathbb{R})=\mathcal{F}_{p}(\mathbb{R})$.

2. $\|h\|_{L^{p}\left(\mathbb{B}_{r}\right)},\|h\|_{L^{q}\left(\mathbb{B}_{r}^{c}\right)}$, and $\|h\|_{L^{2}\left(\mathbb{B}_{r}^{c}\right)}$ are finite for every $h \in \mathcal{F}_{p}\left(\mathbb{R}^{d}\right)$ and $r>0$.

3. If $h \in \mathcal{F}_{p}\left(\mathbb{R}^{d}\right)$, then

$$
\int_{0}^{r} s^{d-1}\left(\|h\|_{L^{p}\left(\mathbb{B}_{s}\right)}\|h\|_{L^{q}\left(\mathbb{B}_{s}^{c}\right)}+\|h\|_{L^{2}\left(\mathbb{B}_{s}^{c}\right)}^{2}\right) \mathrm{d} s<\infty \quad \text { for every } r>0 .
$$

4. If $h \in \mathcal{G}_{p}\left(\mathbb{R}^{d}\right)$, then

$$
\int_{0}^{r}\left(\|h\|_{L^{p}\left(\mathbb{B}_{s}\right)}\|h\|_{L^{q}\left(\mathbb{B}_{s}^{c}\right)}+\|h\|_{L^{2}\left(\mathbb{B}_{s}^{c}\right)}^{2}\right) \omega_{d}(s) \mathrm{d} s<\infty \quad \text { for every } r>0 .
$$

Proof. We have $\mathcal{G}_{p}\left(\mathbb{R}^{d}\right) \subset \mathcal{F}_{p}\left(\mathbb{R}^{d}\right)$ for all $d \geq 2$ and $\mathcal{G}_{p}(\mathbb{R})=\mathcal{F}_{p}(\mathbb{R})$ because of (1.9); and the local integrability of $h \in \mathcal{F}_{p}\left(\mathbb{R}^{d}\right)$ is a consequence of Hölder's inequality. This proves part 1 . We concentrate on the remaining assertions of the lemma.

First, let us note that if $p>1$ and $h \in \mathcal{F}_{p}\left(\mathbb{R}^{d}\right)$, then $h$ is locally in $L^{p}\left(\mathbb{R}^{d}\right)$ and hence $\|h\|_{L^{p}\left(\mathbb{B}_{r}\right)}$ is finite for every $r>0$. In particular,

$$
\|h\|_{L^{q}\left(\mathbb{B}_{r}^{c}\right)}+\|h\|_{L^{2}\left(\mathbb{B}_{r}^{c}\right)}<\infty,
$$

for almost every $r \in[0,1]$. Since both of the norms in (3.4) are monotonically-decreasing functions of $r$, it follows that in fact (3.4) holds for every $r>0$. This proves part 2 of the lemma.

\footnotetext{
${ }^{3}$ Some authors insist that $g$ is of positive type if, in addition to the requirements of Definition $3.1, g(0):=$ $\lim _{x \rightarrow 0} g(x)=\infty$. Others do not insist that $g$ vanishes at infinity.
} 
Next, suppose $r>1$ and observe that

$\int_{1}^{r} s^{d-1}\left(\|h\|_{L^{p}\left(\mathbb{B}_{s}\right)}\|h\|_{L^{q}\left(\mathbb{B}_{s}^{c}\right)}+\|h\|_{L^{2}\left(\mathbb{B}_{s}^{c}\right)}^{2}\right) \mathrm{d} s \leq\left(\|h\|_{L^{p}\left(\mathbb{B}_{r}\right)}\|h\|_{L^{q}\left(\mathbb{B}_{1}^{c}\right)}+\|h\|_{L^{2}\left(\mathbb{B}_{1}^{c}\right)}^{2}\right)\left(\frac{r^{d}-1}{d}\right)$

is finite. This and the definition of the vector space $\mathcal{F}_{p}\left(\mathbb{R}^{d}\right)$ together imply that (3.2) holds; (3.3) is proved similarly.

It follows from local integrability that the Fourier transform of every function $h \in$ $\mathcal{F}_{p}\left(\mathbb{R}^{d}\right)(p>1)$ is a well-defined distribution. In particular, both $f=h * \tilde{h}$ and $|h| *|\tilde{h}|$ are also well-defined distributions. Of course, all such distributions are nonnegativedefinite as well. The following shows that both $h * \tilde{h}$ and $|h| *|\tilde{h}|$ are in fact fairly nice nonnegative-definite functions from $\mathbb{R}^{d}$ to the extended real numbers $\mathbb{R} \cup\{\infty\}$.

Proposition 3.4. If $h \in \mathcal{F}_{p}\left(\mathbb{R}^{d}\right)$ for some $p>1$, then $h * \tilde{h}$ and $|h| *|\tilde{h}|$ are functions of positive type. Moreover, for every $r>0$,

$$
\sup _{\|x\|>2 r}|(h * \tilde{h})(x)| \leq \sup _{\|x\|>2 r}(|h| *|\tilde{h}|)(x) \leq 2\|h\|_{L^{p}\left(\mathbb{B}_{r}\right)}\|h\|_{L^{q}\left(\mathbb{B}_{r}^{c}\right)}+\|h\|_{L^{2}\left(\mathbb{B}_{r}^{c}\right)}^{2},
$$

and

$$
\int_{\mathbb{B}_{r}}(|h| *|\tilde{h}|)(x) \mathrm{d} x \lesssim \int_{0}^{2 r} s^{d-1}\left(\|h\|_{L^{p}\left(\mathbb{B}_{s}\right)}\|h\|_{L^{q}\left(\mathbb{B}_{s}^{c}\right)}+\|h\|_{L^{2}\left(\mathbb{B}_{s}^{c}\right)}^{2}\right) \mathrm{d} s,
$$

where the implied constant depends only on $d$, and $q:=p /(p-1)$.

Proof. The argument hinges loosely on old ideas that are motivated by the literature on potential theory of Lévy processes; see in particular Hawkes [28, 29].

Let us choose and fix arbitrary numbers $r, s>0$ and $x \in \mathbb{R}^{d}$ such that $\|x\|>r+s$. On the one hand, if $y \in \mathbb{B}_{r}$ then certainly $\|x-y\|>s$, whence

$$
\int_{\mathbb{B}_{r}}|h(y) h(y-x)| \mathrm{d} y \leq\|h\|_{L^{p}\left(\mathbb{B}_{r}\right)}\|h\|_{L^{q}\left(\mathbb{B}_{s}^{c}\right)},
$$

by Hölder's inequality. On the other hand, Hölder's inequality ensures that for every $z \in \mathbb{R}^{d}$ and $t>0$,

$$
\begin{aligned}
\int_{\mathbb{B}_{r}^{c}}|h(y) h(y-z)| \mathrm{d} y & \leq \int_{\|y\|>r}|h(y) h(y-z)| \mathrm{d} y+\int_{\|y\| y \|>r}|h(y) h(y-z)| \mathrm{d} y \\
& \leq\|h\|_{L^{p}\left(\mathbb{B}_{t}\right)}\|h\|_{L^{q}\left(\mathbb{B}_{r}^{c}\right)}+\|h\|_{L^{2}\left(\mathbb{B}_{r}^{c}\right)}\|h\|_{L^{2}\left(\mathbb{B}_{t}^{c}\right)} .
\end{aligned}
$$

Combine the above bounds to find that

$$
\sup _{\|x\|>r+s}(|h| *|\tilde{h}|)(x) \leq\|h\|_{L^{p}\left(\mathbb{B}_{r}\right)}\|h\|_{L^{q}\left(\mathbb{B}_{s}^{c}\right)}+\|h\|_{L^{p}\left(\mathbb{B}_{t}\right)}\|h\|_{L^{q}\left(\mathbb{B}_{r}^{c}\right)}+\|h\|_{L^{2}\left(\mathbb{B}_{r}^{c}\right)}\|h\|_{L^{2}\left(\mathbb{B}_{t}^{c}\right)},
$$

for every $r, s, t>0$. If $h \in \mathcal{F}_{p}\left(\mathbb{R}^{d}\right)$ then certainly $|h| \in \mathcal{F}_{p}\left(\mathbb{R}^{d}\right)$ also, and we can set $s=r=t$ in order to deduce (3.5) from (3.7). Also, we may first let $s \rightarrow \infty$, and then let $r \rightarrow \infty$ in (3.7) - in this order - to see that $|h| *|\tilde{h}|$ vanishes at infinity.

Next, we verify (3.6) by merely observing that

$$
\int_{\mathbb{B}_{r}}(|h| *|\tilde{h}|)(x) \mathrm{d} x \leq \int_{\mathbb{B}_{r}} \Phi(\|x\| / 2) \mathrm{d} x \propto \int_{0}^{2 r} \Phi(s) s^{d-1} \mathrm{~d} s,
$$

where $\Phi(t):=\sup _{\|x\|>2 t}(|h| *|\tilde{h}|)(x)$ for every $t>0$. Apply the already-proved part of the lemma, together with Lemma 3.3, in order to see that $|h| *|\tilde{h}| \in L_{\text {loc }}^{1}\left(\mathbb{R}^{d}\right)$. 
The same argument that led to (3.7) (with $r=s=t$ ) yields that $\sup _{\|x\|>2 r}\left(\left|h_{1}\right| *\left|\tilde{h}_{2}\right|\right)(x) \leq\left\|h_{1}\right\|_{L^{p}\left(\mathbb{B}_{r}\right)}\left\|h_{2}\right\|_{L^{q}\left(\mathbb{B}_{r}^{c}\right)}+\left\|h_{2}\right\|_{L^{p}\left(\mathbb{B}_{r}\right)}\left\|h_{1}\right\|_{L^{q}\left(\mathbb{B}_{r}^{c}\right)}+\left\|h_{1}\right\|_{L^{2}\left(\mathbb{B}_{r}^{c}\right)}\left\|h_{2}\right\|_{L^{2}\left(\mathbb{B}_{r}^{c}\right)}$,

whenever $h_{1}, h_{2} \in \mathcal{F}_{p}\left(\mathbb{R}^{d}\right)$. Choose and fix an approximation to the identity $\left\{\varphi_{\varepsilon}\right\}_{\varepsilon>0}$ such that $\varphi_{\varepsilon} \in C_{c}^{\infty}\left(\mathbb{R}^{d}\right)$ for every $\varepsilon>0$. We may apply the preceding displayed inequality, once with $\left(h_{1}, h_{2}\right)=\left(h, h-\left(\varphi_{\varepsilon} * h\right)\right)$ and once with $\left(h_{1}, h_{2}\right)=\left(|h|,|h|-\left(\varphi_{\varepsilon} *|h|\right)\right)$, in order to see that as $\varepsilon \downarrow 0,\left(\varphi_{\varepsilon} *|h| *|\tilde{h}|\right)(x) \rightarrow(|h| *|\tilde{h}|)(x)$ and $\left(\varphi_{\varepsilon} * h * \tilde{h}\right)(x) \rightarrow(h * \tilde{h})(x)$, both valid uniformly for all $x \in \mathbb{R}^{d}$ that satisfy $\|x\|>2 r$. This uses only the classical fact that

$$
\lim _{\varepsilon \downarrow 0}\left(\left\|g-\left(\varphi_{\varepsilon} * g\right)\right\|_{L^{p}\left(\mathbb{B}_{r}\right)}+\left\|g-\left(\varphi_{\varepsilon} * g\right)\right\|_{L^{q}\left(\mathbb{B}_{r}^{c}\right)}+\left\|g-\left(\varphi_{\varepsilon} * g\right)\right\|_{L^{2}\left(\mathbb{B}_{r}^{c}\right)}\right)=0,
$$

for either $g=h$ or $g=|h|$ (see Stein [52]), and readily implies the uniform continuity and boundedness of $h * \tilde{h}$ and $|h| *|\tilde{h}|$ off $\mathbb{B}_{r}$ for arbitrary $r>0$. This completes the proof that $h * \tilde{h}$ and $|h| *|\tilde{h}|$ are functions of positive type.

\subsection{On Condition (1.4)}

As was mentioned in the Introduction, it was shown by Dalang [19] that when $f$ is tempered and non negative, Condition (1.4) is an optimal sufficient condition for the existence of a unique random-field solution to the SPDE (1.1). In this section, we say a few words about Dalang's Condition (1.4) in the setting where $f$ is a function that satisfies (1.8).

First recall that the vector space $H_{-1}\left(\mathbb{R}^{d}\right)$ denotes the completion of all rapidlydecreasing, real-valued $C^{\infty}$-functions on $\mathbb{R}^{d}$ in the norm

$$
\|h\|_{H_{-1}\left(\mathbb{R}^{d}\right)}:=\left(\int_{\mathbb{R}^{d}} \frac{|\hat{h}(x)|^{2}}{1+\|x\|^{2}} \mathrm{~d} x\right)^{1 / 2} .
$$

It follows immediately that $H_{-1}\left(\mathbb{R}^{d}\right)$ is Hilbertian, once endowed with the above norm and the associated inner product,

$$
\left\langle\psi_{1}, \psi_{2}\right\rangle_{H_{-1}\left(\mathbb{R}^{d}\right)}:=\int_{\mathbb{R}^{d}} \frac{\hat{\psi}_{1}(x) \overline{\hat{\psi}_{2}(x)}}{1+\|x\|^{2}} \mathrm{~d} x .
$$

Next, let us define $\boldsymbol{v}_{\lambda}$ to be the $\lambda$-potential density of the heat semigroup on $\mathbb{R}^{d}$ for every $\lambda>0$. That is,

$$
\boldsymbol{v}_{\lambda}(x)=\int_{0}^{\infty} \mathrm{e}^{-\lambda t} \boldsymbol{p}_{t}(x) \mathrm{d} t \quad \text { for all } x \in \mathbb{R}^{d},
$$

where $\boldsymbol{p}$ denotes the heat kernel, defined as

$$
\boldsymbol{p}_{t}(x):=\frac{1}{(2 \pi t)^{d / 2}} \exp \left(-\frac{\|x\|^{2}}{2 t}\right) \quad \text { for all } t>0 \text { and } x \in \mathbb{R}^{d} .
$$

Note that $\lambda \boldsymbol{v}_{\lambda}$ is a probability density function on $\mathbb{R}^{d}$ for every $\lambda>0$.

A general theorem of Foondun and Khoshnevisan [26] implies that when $f=h * \tilde{h}$ is a function and $h \geq 0$ (and hence $f \geq 0$ ), Dalang's condition (1.4) holds if and only if ${ }^{4}$

$$
\int_{\mathbb{R}^{d}} \boldsymbol{v}_{\lambda}(x) f(x) \mathrm{d} x<\infty \text { for one, hence all, } \lambda>0 .
$$

\footnotetext{
${ }^{4}$ In general, the proof of (3.10) requires some effort. But, for example when $h \in L^{1}\left(\mathbb{R}^{d}\right) \cap L^{2}\left(\mathbb{R}^{d}\right)$, Young's inequality yields $f \in \cap_{\nu \in[1, \infty]} L^{\nu}\left(\mathbb{R}^{d}\right)$ and hence (3.10) is a direct consequence of Parseval's identity and the elementary facts that: (i) The Fourier transform of $\boldsymbol{v}_{\lambda}$ is $\hat{\boldsymbol{v}}_{\lambda}(z):=\int_{\mathbb{R}^{d}} \exp \{i x \cdot z\} \boldsymbol{v}_{\lambda}(x) \mathrm{d} x=2\left[2 \lambda+\|z\|^{2}\right]^{-1}$ for all $z \in \mathbb{R}^{d}$; and (ii) $\hat{f}(z):=\int_{\mathbb{R}^{d}} \exp \{i x \cdot z\} f(x) \mathrm{d} x=|\hat{h}(z)|^{2}$ for all $z \in \mathbb{R}^{d}$.
} 
[To use the results of [26] we need also the easy-to-prove fact that $h * \tilde{h}$ is lower semicontinuous in the present setting.] An earlier result, applicable in the present context, can be found in Peszat [49, Theorem 0.1].

Let us note also that if $h \geq 0$ and $h \in \mathcal{F}_{p}\left(\mathbb{R}^{d}\right)$ for some $p>1$, then $f$ is bounded uniformly on $\mathbb{B}_{r}^{c}$ for all $r>0$. Because in addition $\boldsymbol{v}_{\lambda}$ is integrable, it follows from (3.10) that, in the present setting wherein $h \geq 0$ and $h \in \cup_{p>1} \mathcal{F}_{p}\left(\mathbb{R}^{d}\right)$, the harmonic-analytic condition (1.4)—equivalently the potential-theoretic condition (3.10) - is equivalent to the following local version of (3.10):

$$
\int_{\mathbb{B}_{1}} \boldsymbol{v}_{\lambda}(x) f(x) \mathrm{d} x<\infty \quad \text { for one, hence all, } \lambda>0 .
$$

Next, we re-interpret (3.11): It is well known, and easy to verify directly (see, for example, [38, Chapter 10, Section 3.1]), that

$$
\boldsymbol{v}_{\lambda}(x) \asymp\|x\|^{-d+1} \omega_{d}(\|x\|) \quad \text { uniformly for all } x \in \mathbb{B}_{1},
$$

where $\omega_{d}$ was defined in (1.9). Thus, when $h \geq 0$ and $h \in \cup_{p>1} \mathcal{F}_{p}\left(\mathbb{R}^{d}\right)$,

$$
h \in H_{-1}\left(\mathbb{R}^{d}\right) \quad \text { iff } \quad \int_{\mathbb{B}_{1}}\|x\|^{-d+1} \omega_{d}(\|x\|) f(x) \mathrm{d} x<\infty .
$$

Next let us consider the general case where $h \in \cup_{p>1} \mathcal{F}_{p}\left(\mathbb{R}^{d}\right)$ is possibly signed. Since $|f(x)| \leq(|h| *|\tilde{h}|)(x)$ for all $x \in \mathbb{R}^{d}$, we can apply (3.13) with $(h, f)$ replaced with $(|h|,|h| *|\tilde{h}|)$ in order to see that

$$
\text { if } \int_{0}^{1} \sup _{\|y\|>r}(|h| *|\tilde{h}|)(y) \omega_{d}(r) \mathrm{d} r<\infty, \quad \text { then }|h| \in H_{-1}\left(\mathbb{R}^{d}\right) .
$$

If $f \geq 0$ and $x \mapsto f(x)$ is a radial function on $\mathbb{R}^{d}$ that decreases as $\|x\|$ increases, then $\sup _{\|y\|>\|x\|}(|h| *|\tilde{h}|)(y)=f(x)$, and the above sufficient condition for $|h|=h$ to be in $H_{-1}\left(\mathbb{R}^{d}\right)$ appears earlier in the literature, in the context of well-posedness for SPDEs. See Dalang and Frangos [20], Karczewska and Zabczyk [36], Peszat [49], and Peszat and Zabczyk [50]. Closely-related results can be found in Cardon-Weber and Millet [6], Dalang [19], Foondun and Khoshnevisan [26], and Millet and Sanz-Solé [43].

Recall the vector space $\cup_{p>1} \mathcal{G}_{p}\left(\mathbb{R}^{d}\right)$ (Definition 3.2) and the inequalities of Proposition 3.4 in order to deduce the following.

Lemma 3.5. If $h \in \mathcal{G}_{p}\left(\mathbb{R}^{d}\right)$ for some $p>1$, then $|h| \in H_{-1}\left(\mathbb{R}^{d}\right)$. In particular, $h \in$ $\cup_{p>1} \mathcal{G}_{p}\left(\mathbb{R}^{d}\right)$ implies that $\int_{\mathbb{R}^{d}} \boldsymbol{v}_{\lambda}(x)(|h| *|\tilde{h}|)(x) \mathrm{d} x<\infty$ for some, hence all, $\lambda>0$.

In light of Theorem 1.2 of Foondun and Khoshnevisan [26], Lemma 3.5 implies a precise version of the somewhat subtle assertion that sufficient integrability of $h$ ensures good decay at infinity of the Fourier transform of $|h|$.

\subsection{Proof of part 2 of Theorem 1.1}

In this section, we prove part 2 of Theorem 1.1. In fact, the work involves proving the following harmonic-analytic result.

Proposition 3.6. Suppose $f \in \mathfrak{M}_{+}\left(\mathbb{R}^{d}\right)$; that is, $f$ is a nonnegative-definite tempered Borel measure on $\mathbb{R}^{d}$. Suppose, in addition, that

$$
\int_{\mathbb{R}^{d}} \prod_{j=1}^{d}\left(\frac{1}{1 \vee y_{j}^{2}}\right) \hat{f}(\mathrm{~d} y)<\infty .
$$

Then, $\hat{f}(\{0\})=0$ iff $f\left(\mathbb{B}_{r}\right)=o\left(r^{d}\right)$ as $r \rightarrow \infty$. 


\section{Spatial ergodicity for SPDEs}

Proposition 3.6 readily implies part 2 of Theorem 1.1 since (1.4) implies (3.14). Therefore, it remains to prove Proposition 3.6.

Proof of Proposition 3.6. Consider, for every real number $N>0$, the probability density function

$$
I_{N}:=N^{-d} \mathbf{1}_{[0, N]^{d}} \quad \text { on } \mathbb{R}^{d} .
$$

Then,

$$
\left(I_{N} * \tilde{I}_{N}\right)(x)=N^{-d} \prod_{j=1}^{d}\left(1-\frac{\left|x_{j}\right|}{N}\right)_{+} \quad \text { for every } x=\left(x_{1}, \ldots, x_{d}\right) \in \mathbb{R}^{d},
$$

where $a_{+}:=\max \{a, 0\}$. Because $\frac{1}{2} \mathbf{1}_{[-1 / 2,1 / 2]}(a) \leq(1-|a|)_{+} \leq \mathbf{1}_{[-1,1]}(a)$ for every $a \in \mathbb{R}$,

$$
(2 N)^{-d} \mathbf{1}_{[-N / 2, N / 2]^{d}} \leq I_{N} * \tilde{I}_{N} \leq N^{-d} \mathbf{1}_{[-N, N]^{d}} \quad \text { on } \mathbb{R}^{d} .
$$

Since $I_{N} * \tilde{I}_{N} \in C_{c}\left(\mathbb{R}^{d}\right)$ and the measure $f$ is locally finite, it follows that $I_{N} * \tilde{I}_{N} * f$ is continuous and bounded. Because $I_{N} * \tilde{I}_{N} * f$ is also nonnegative definite, it is therefore maximized at 0 . These properties, and the well-known fact that $\left\{\boldsymbol{p}_{t}\right\}_{t>0}$ is a (convolution) Feller semigroup, together imply that

$$
\left(I_{N} * \tilde{I}_{N} * f\right)(0)=\lim _{\varepsilon \rightarrow 0}\left(I_{N} * \tilde{I}_{N} * \boldsymbol{p}_{\varepsilon} * f\right)(0),
$$

where $\boldsymbol{p}_{\varepsilon}$ denotes the Gaussian heat kernel of (3.9). We may apply Parseval's formula (1.3) next in order to see that

$$
\left(\boldsymbol{p}_{\varepsilon} * f\right)(x)=\int_{\mathbb{R}^{d}} \exp \left(i x \cdot y-\frac{\varepsilon}{2}\|y\|^{2}\right) \hat{f}(\mathrm{~d} y) \quad \text { for every } x \in \mathbb{R}^{d} \text { and } \varepsilon>0 .
$$

Therefore, Tonelli's theorem readily yields the identity

$$
\left(I_{N} * \tilde{I}_{N} * \boldsymbol{p}_{\varepsilon} * f\right)(0)=2^{d} \int_{\mathbb{R}^{d}} \hat{f}(\mathrm{~d} y) \mathrm{e}^{-\varepsilon\|y\|^{2} / 2} \prod_{j=1}^{d} \frac{1-\cos \left(N y_{j}\right)}{\left(N y_{j}\right)^{2}},
$$

where $2[1-\cos 0] / 0^{2}:=1$. Let $\varepsilon \downarrow 0$ and appeal to the monotone convergence theorem in order to arrive at the identity

$$
\left(I_{N} * \tilde{I}_{N} * f\right)(0)=2^{d} \int_{\mathbb{R}^{d}} \hat{f}(\mathrm{~d} y) \prod_{j=1}^{d} \frac{1-\cos \left(N y_{j}\right)}{\left(N y_{j}\right)^{2}} .
$$

Because $\prod_{j=1}^{d}\left[1-\cos \left(a_{j}\right)\right] / a_{j}^{2} \leq 2^{-d} \prod_{j=1}^{d} \min \left(1, a_{j}^{-2}\right)$ for all $a=\left(a_{1}, \ldots, a_{d}\right) \in \mathbb{R}^{d} \backslash\{0\}$, the dominated convergence theorem and (3.14) together ensure that $\left(I_{N} * \tilde{I}_{N} * f\right)(0)$ converges to $\hat{f}(\{0\})$ as $N \rightarrow \infty$. Thus, we may deduce from (3.17) that

$$
2^{-d} \limsup _{N \rightarrow \infty} \frac{f\left([-N, N]^{d}\right)}{(2 N)^{d}} \leq \hat{f}(\{0\}) \leq 2^{d} \liminf _{N \rightarrow \infty} \frac{f\left([-N, N]^{d}\right)}{(2 N)^{d}} .
$$

Because $\left|\mathbb{B}_{N}\right| \propto N^{d}$ and $\mathbb{B}_{N} \subset[-N, N]^{d} \subset \mathbb{B}_{N \sqrt{d}}$, the above inequalities imply that $\hat{f}(\{0\})=0$ if and only if $f\left(\mathbb{B}_{N}\right)=o\left(\left|\mathbb{B}_{N}\right|\right)$ as $N \rightarrow \infty$. 


\subsection{Proof of part $4 \mathrm{of}$ Theorem 1.1}

Since $\hat{f} \in \mathfrak{M}_{+}\left(\mathbb{R}^{d}\right)$, one can see easily that $I_{N} * \tilde{I}_{N} * \hat{f}$ is a continuous, nonnegativedefinite function for every $N>0$, where $I_{N}$ was defined in (3.15). In particular,

$$
\left(I_{N} * \tilde{I}_{N} * \hat{f}\right)(x) \leq\left(I_{N} * \tilde{I}_{N} * \hat{f}\right)(0) \quad \text { for every } x \in \mathbb{R}^{d} \text { and } N>0 .
$$

Multiply both sides by $N^{d}$ and let $N \rightarrow 0$ in order to deduce from (3.16) and the dominated convergence theorem, $\hat{f}(\{x\}) \leq \hat{f}(\{0\})$ for every $x \in \mathbb{R}^{d}$. This completes the proof.

\section{Proof of part 3 of Theorem 1.1}

In the previous section we verified part 2 of Theorem 1.1. Now we establish the third part of that theorem. Part 1 will be proved a few sections hence.

Suppose there exists a number $c_{0} \in \mathbb{R} \backslash\{0\}$ such that $\sigma(x)=c_{0}$ for all $x \in \mathbb{R}$. In this case, the solution to (1.1) can be written, in mild form, as

$$
u(t, x)=1+c_{0} \int_{(0, t) \times \mathbb{R}^{d}} \boldsymbol{p}_{t-s}(x-z) \eta(\mathrm{d} s \mathrm{~d} z) .
$$

We see from this that, among other things, $u(t)$ is a stationary, mean-one Gaussian random field. Dalang's theory [19] ensures that $x \mapsto u(t, x)$ in continuous in $L^{2}(\Omega)$ for every $t>0$. Therefore, $x \mapsto u(t, x)$ has a Lebesgue-measurable version (which we continue to write as $x \mapsto u(t, x)$ ); see Remark 5.5.

Because of (4.1),

$$
\begin{aligned}
& \int_{[0, N]^{d}} \mathrm{~d} x \int_{[0, N]^{d}} \mathrm{~d} y \operatorname{Cov}(u(t, x), u(t, y)) \\
& =c_{0}^{2} \int_{0}^{t} \mathrm{~d} s \int_{[0, N]^{d}} \mathrm{~d} x \int_{[0, N]^{d}} \mathrm{~d} y\left\langle\boldsymbol{p}_{s}(x-\bullet),\left(\boldsymbol{p}_{s}(y-\bullet)\right) * f\right\rangle_{L^{2}\left(\mathbb{R}^{d}\right)} \quad \text { [by (1.2)] } \\
& =c_{0}^{2} \int_{0}^{t} \mathrm{~d} s \int_{[0, N]^{d}} \mathrm{~d} x \int_{[0, N]^{d}} \mathrm{~d} y \int_{\mathbb{R}^{d}} \hat{f}(\mathrm{~d} z) \mathrm{e}^{i z \cdot(x-y)-s\|z\|^{2}}
\end{aligned}
$$

thanks to Parseval's identity (1.3). Rearrange the integrals, using Fubini's theorem, and compute directly in order to find that

$$
\int_{[0, N]^{d}} \mathrm{~d} x \int_{[0, N]^{d}} \mathrm{~d} y \operatorname{Cov}(u(t, x), u(t, y))=2^{d} c_{0}^{2} \int_{\mathbb{R}^{d}} \hat{f}(\mathrm{~d} z) \frac{1-\mathrm{e}^{-t\|z\|^{2}}}{\|z\|^{2}} \prod_{j=1}^{d} \frac{1-\cos \left(N z_{j}\right)}{z_{j}^{2}},
$$

where $2[1-\cos 0] / 0^{2}:=1$. Since $f$ satisfies Dalang's condition (1.4), the dominated convergence theorem implies that

$$
\lim _{N \rightarrow \infty} \frac{1}{N^{2 d}} \int_{[0, N]^{d}} \mathrm{~d} x \int_{[0, N]^{d}} \mathrm{~d} y \operatorname{Cov}(u(t, x), u(t, y))=c_{0}^{2} t \hat{f}(\{0\}) .
$$

Now suppose, in addition, that $u$ is spatially ergodic. Because $\mathrm{E}[u(t, x)]=1$ (see (4.1)), von Neumann's mean ergodic theorem (see for example Peterson [51], and especially Chapters 8 and $\$ 9.3$ of Edgar and Sucheston [24]) implies that $N^{-d} \int_{[0, N]^{d}} u(t, x) \mathrm{d} x$ converges in $L^{2}(\Omega)$ to 1 as $N \rightarrow \infty$. Equivalently, that

$$
\lim _{N \rightarrow \infty} \frac{1}{N^{2 d}} \int_{[0, N]^{d}} \mathrm{~d} x \int_{[0, N]^{d}} \mathrm{~d} y \operatorname{Cov}(u(t, x), u(t, y))=0 .
$$

Part 3 of Theorem 1.1 follows from comparing (4.2) and (4.3). 


\section{Well posedness}

By Dalang [19], equation (1.1) is well-posed when the spatial correlation $f$ satisfies condition (1.4). In this section, we only prove the well posedness of (1.1) when $f=h * \tilde{h}$ with $h \in \cup_{p>1} \mathcal{G}_{p}\left(\mathbb{R}^{d}\right)$.

\subsection{Stochastic convolutions}

If $\Phi=\{\Phi(t, x)\}_{t \geq 0, x \in \mathbb{R}^{d}}$ is a space-time random field, then for all real numbers $\beta>0$ and $k \geq 1$, we may define

$$
\mathcal{N}_{\beta, k}(\Phi):=\sup _{t \geq 0} \sup _{x \in \mathbb{R}^{d}} \mathrm{e}^{-\beta t}\|\Phi(t, x)\|_{k}
$$

It is clear that $\Phi \mapsto \mathcal{N}_{\beta, k}(\Phi)$ defines a norm for every choice of $\beta>0$ and $k \geq 1$. These norms were first introduced in [25]; see also [16]. Corresponding to every $\mathcal{N}_{\beta, k}$, define $\mathrm{W}_{\beta, k}$ to be the collection of all predictable random fields $\Phi$ such that $\mathcal{N}_{\beta, k}(\Phi)<\infty$. We may think of elements of $W_{\beta, 2}$ as Walsh-integrable random fields with Lyapunov exponent $\leq \beta$. It is easy to see that each $\left(\mathbb{W}_{\beta, k}, \mathcal{N}_{\beta, k}\right)$ is a Banach space; see [37].

Suppose that the underlying probability space $(\Omega, \mathcal{F}, \mathrm{P})$ is large enough to carry a space-time white noise $\xi$ (if not then enlarge it in the usual way). Using that noise, we may formally define, for every fixed measurable function $h: \mathbb{R}^{d} \rightarrow \mathbb{R}$, a new noise $\eta^{(h)}$ as follows:

$$
\eta^{(h)}(\mathrm{d} s \mathrm{~d} x):=\int_{\mathbb{R}^{d}} h(x-y) \xi(\mathrm{d} s \mathrm{~d} y) \mathrm{d} x .
$$

Somewhat more precisely, if $H$ is a predictable random field such that

$$
\mathrm{E} \int_{0}^{t} \mathrm{~d} s \int_{\mathbb{R}^{d}} \mathrm{~d} y|(H(s) * \tilde{h})(y)|^{2}<\infty \quad \text { for every } t>0,
$$

then Walsh's theory of stochastic integration ensures that the Walsh stochastic integral

$$
\int_{(0, t) \times \mathbb{R}^{d}} H(s, x) \eta^{(h)}(\mathrm{d} s \mathrm{~d} x):=\int_{(0, t) \times \mathbb{R}^{d}}(H(s) * \tilde{h})(y) \xi(\mathrm{d} s \mathrm{~d} y)
$$

is well-defined for every $t \geq 0$, and in fact defines a continuous, mean-zero, $L^{2}(\Omega)$ martingale indexed by $t \geq 0$. Moreover, the variance of this martingale at time $t>0$ is

$$
\begin{aligned}
\mathrm{E}\left(\left|\int_{(0, t) \times \mathbb{R}^{d}} H(s, x) \eta^{(h)}(\mathrm{d} s \mathrm{~d} x)\right|^{2}\right) & =\mathrm{E} \int_{0}^{t} \mathrm{~d} s \int_{\mathbb{R}^{d}} \mathrm{~d} y|(H(s) * \tilde{h})(y)|^{2} \\
& =\int_{0}^{t} \mathrm{~d} s \int_{\mathbb{R}^{d}} \mathrm{~d} y \int_{\mathbb{R}^{d}} \mathrm{~d} z \mathrm{E}[H(s, y) H(s, z)] f(y-z),
\end{aligned}
$$

provided, for example, that the preceding integral is absolutely convergent. (As it is the case, here and elsewhere in this section, $f$ is defined in terms of $h \in \cup_{p>1} \mathcal{G}_{p}\left(\mathbb{R}^{d}\right)$ via (1.8).)

It is easy to see from this that $\eta^{(h)}$ is a particular construction of the noise $\eta$ of the Introduction (see also Conus et al [18]), but has the advantage that it provides a coupling $h \mapsto \eta^{(h)}$ that works simultaneously for many different choices of $h$, whence of spatial correlation functions $f$.

The preceding stochastic integration (see (5.3)) frequently allows for the integration of a large family of predictable random fields $H$. The following simple result highlights a large subclass of such random fields when $h \in \cup_{p>1} \mathcal{F}_{p}\left(\mathbb{R}^{d}\right)$. 
Lemma 5.1. Suppose $h \in \mathcal{F}_{p}\left(\mathbb{R}^{d}\right)$ for some $p>1$, and $H$ is a predictable process for which there exists a real number $r>0$ such that

$$
\sup _{s \in[0, T]} \sup _{y \in \mathbb{R}^{d}} \mathrm{E}\left(|H(s, y)|^{2}\right)<\infty \quad \text { and } \mathrm{E}\left(|H(t, x)|^{2}\right)=0 \quad \text { for every } t>0 \text { and } x \in \mathbb{B}_{r}^{c} .
$$

Then the final integral in (5.3) is absolutely convergent and hence (5.3) is valid for every $t>0$.

Proof. Choose and fix an arbitrary $t>0$. Thanks to the Cauchy-Schwarz inequality, it suffices to prove that

$$
J:=\int_{0}^{t} \mathrm{~d} s \int_{\mathbb{B}_{r}} \mathrm{~d} y \int_{\mathbb{B}_{r}} \mathrm{~d} z\|H(s, y)\|_{2}\|H(s, z)\|_{2}|f(y-z)|<\infty \quad \text { for every } t>0 .
$$

But the triangle inequality readily yields

$$
J \leq\left|\mathbb{B}_{r}\right|\left(\int_{0}^{t} \sup _{y \in \mathbb{R}^{d}}\|H(s, y)\|_{2}^{2} \mathrm{~d} s\right)\left(\int_{\mathbb{B}_{2 r}}(|h| *|\tilde{h}|)(w) \mathrm{d} w\right),
$$

which is finite thanks to (5.4) and Proposition 3.4; see in particular (3.6).

The second portion of (5.4) involves a compact-support condition which can sometimes be reduced to a decay-type condition. We exemplify that next for a specific family of the form $H(s, y)=\boldsymbol{p}_{t-s}(x-y) Z(s, y)$, where $t>s$ and $x \in \mathbb{R}^{d}$ are fixed and $\boldsymbol{p}$ denotes the heat kernel [see (3.9)]. With this choice, the following "stochastic convolution" is a well-defined random field provided that it is indeed defined properly as a Walsh integral for every $t>0$ and $x \in \mathbb{R}^{d}$ :

$$
\left(\boldsymbol{p} \circledast Z \eta^{(h)}\right)(t, x):=\int_{(0, t) \times \mathbb{R}^{d}} \boldsymbol{p}_{t-s}(x-y) Z(s, y) \eta^{(h)}(\mathrm{d} s \mathrm{~d} y) .
$$

For every $k \geq 2$, let $z_{k}^{k}$ denote the optimal constant of the $L^{k}(\Omega)$-form of the Burkholder-Davis-Gundy inequality [2, 3, 4]; that is, for every continuous $L^{2}(\Omega)$-martingale $\left\{M_{t}\right\}_{t \geq 0}$, and all real numbers $k \geq 2$ and $t \geq 0$,

$$
\mathrm{E}\left(\left|M_{t}\right|^{k}\right) \leq z_{k}^{k} \mathrm{E}\left(\langle M\rangle_{t}^{k / 2}\right)
$$

Then,

$$
z_{2}=1 \quad \text { and } \quad z_{k} \leq 2 \sqrt{k} \quad \text { for every } k>2 .
$$

The first assertion is the basis of Itô's stochastic calculus, and the second is due to Carlen and Kree [7], who also proved that $\lim _{k \rightarrow \infty}\left(z_{k} / \sqrt{k}\right)=2$. The exact value of $z_{k}$ is computed in the celebrated paper of Davis [21].

The following provides a natural condition for the stochastic convolution to be a well-defined random field, the stochastic integral being defined in the sense of Walsh [53], and extends Proposition 6.1 of Conus et al [18] to the case that $f$ is possibly signed. It might help to recall that $\boldsymbol{v}_{\beta}$ denotes the $\beta$-potential kernel [see (3.8)].

Lemma 5.2 (A stochastic Young inequality). Suppose that $Z \in \mathbb{W}_{\beta, k}$ for some $\beta>0$, $k \geq 2$, and that $h \in \mathcal{G}_{p}\left(\mathbb{R}^{d}\right)$ for some $p>1$. Then the stochastic convolution in (5.5) is a well-defined Walsh integral,

$$
\mathcal{N}_{\beta, k}\left(\boldsymbol{p} \circledast Z \eta^{(h)}\right) \leq z_{k} \mathcal{N}_{\beta, k}(Z) \sqrt{\frac{1}{2} \int_{\mathbb{R}^{d}} \boldsymbol{v}_{\beta}(x)|f(x)| \mathrm{d} x,}
$$

and the integral under the square root is finite. 
Proof. The integral under the square root is finite thanks to Lemma 3.5. We proceed to prove the remainder of the lemma. According to the theory of Walsh [53], the random field $\boldsymbol{p} \circledast Z \eta^{(h)}$ is well defined whenever $\mathcal{Q}_{2}(t, x)<\infty$ where

$$
\mathcal{Q}_{\kappa}(t, x):=\int_{0}^{t} \mathrm{~d} s \int_{\mathbb{R}^{d}} \mathrm{~d} y \int_{\mathbb{R}^{d}} \mathrm{~d} z \boldsymbol{p}_{t-s}(x-y) \boldsymbol{p}_{t-s}(x-z)\|Z(s, y)\|_{k}\|Z(s, z)\|_{k}|f(y-z)|
$$

for every $t>0$ and $x \in \mathbb{R}^{d}$. Moreover (see also (5.3)), in that case, the Burkholder-DavisGundy inequality yields

$$
\begin{aligned}
& \mathrm{E}\left(\left|\left(\boldsymbol{p} \circledast Z \eta^{(h)}\right)(t, x)\right|^{k}\right) \\
& \leq z_{k}^{k} \mathrm{E}\left(\left|\int_{0}^{t} \mathrm{~d} s \int_{\mathbb{R}^{d}} \mathrm{~d} y \int_{\mathbb{R}^{d}} \mathrm{~d} z \boldsymbol{p}_{t-s}(x-y) \boldsymbol{p}_{t-s}(x-z) Z(s, y) Z(s, z) f(y-z)\right|^{k / 2}\right) \\
& \leq z_{k}^{k}\left[\int_{0}^{t} \mathrm{~d} s \int_{\mathbb{R}^{d}} \mathrm{~d} y \int_{\mathbb{R}^{d}} \mathrm{~d} z \boldsymbol{p}_{t-s}(x-y) \boldsymbol{p}_{t-s}(x-z)\|Z(s, y) Z(s, z)\|_{k / 2}|f(y-z)|\right]^{k / 2} \\
& \leq z_{k}^{k}\left[\mathcal{Q}_{\kappa}(t, x)\right]^{k / 2},
\end{aligned}
$$

the last line holding thanks to the Cauchy-Schwarz inequality. It remains to prove that $\mathcal{Q}_{\kappa}(t, x)<\infty$ for all $t>0$ and $x \in \mathbb{R}^{d}$.

Since $\|Z(s, y)\|_{k} \leq \exp (\beta s) \mathcal{N}_{\beta, k}(Z)$ for all $s \geq 0$ and $y \in \mathbb{R}^{d}$, it then follows that

$$
\begin{aligned}
\mathcal{Q}_{\kappa}(t, x) & \leq\left[\mathcal{N}_{\beta, k}(Z)\right]^{2} \int_{0}^{t} \mathrm{e}^{-2 \beta s} \mathrm{~d} s \int_{\mathbb{R}^{d}} \mathrm{~d} y \int_{\mathbb{R}^{d}} \mathrm{~d} z \boldsymbol{p}_{t-s}(x-y) \boldsymbol{p}_{t-s}(x-z)|f(y-z)| \\
& \leq \mathrm{e}^{2 \beta t}\left[\mathcal{N}_{\beta, k}(Z)\right]^{2} \int_{0}^{t} \mathrm{e}^{-2 \beta r} \mathrm{~d} r \int_{\mathbb{R}^{d}} \mathrm{~d} w \boldsymbol{p}_{2 r}(w)|f(w)|,
\end{aligned}
$$

after two change of variables $[w=y-z, r=t-s]$, and thanks to the ChapmanKolmogorov (semigroup) property of the heat kernel $\boldsymbol{p}$. Since

$$
\int_{0}^{t} \exp (-2 \beta r) \boldsymbol{p}_{2 r}(w) \mathrm{d} r \leq \int_{0}^{\infty} \exp (-2 \beta r) \boldsymbol{p}_{2 r}(w) \mathrm{d} r=\frac{1}{2} \boldsymbol{v}_{\beta}(w),
$$

for every $w \in \mathbb{R}^{d}$ and $\beta>0$, this proves that

$$
\mathrm{e}^{-2 \beta t} \mathcal{Q}_{\kappa}(t, x) \leq \frac{1}{2}\left[\mathcal{N}_{\beta, k}(Z)\right]^{2} \int_{\mathbb{R}^{d}} \boldsymbol{v}_{\beta}(w)|f(w)| .
$$

This inequality completes the proof of the lemma upon taking square roots, as the right-hand side of the preceding inequality is independent of $(t, x)$.

\subsection{Well posedness}

Before we study the spatial ergodicity of the solution to (1.1) we address matters of well posedness. As was mentioned earlier, well-posedness follows from the more general theory of Dalang [19] when $h \geq 0$, for example. Here we say a few things about general well posedness when $h$ is signed. This undertaking does require some new ideas, but most of those new ideas have already been developed in the earlier sections, particularly as regards the space $\cup_{p>1} \mathcal{G}_{p}\left(\mathbb{R}^{d}\right)$, which now plays a prominent role.

Recall the $\lambda$-potential $\boldsymbol{v}_{\lambda}$ from (3.8). Choose and fix a function $h \in \cup_{p>1} \mathcal{G}_{p}\left(\mathbb{R}^{d}\right)$ and recall from Lemma 3.5 that

$$
\int_{\mathbb{R}^{d}} \boldsymbol{v}_{\lambda}(x)|f(x)| \mathrm{d} x \leq \int_{\mathbb{R}^{d}} \boldsymbol{v}_{\lambda}(x)(|h| *|\tilde{h}|)(x) \mathrm{d} x<\infty,
$$


for one, hence all, $\lambda>0$. As a consequence, we find that the following is a well-defined, $(0, \infty)$-valued function on $(0, \infty)$ :

$$
\Lambda_{h}(\delta):=\inf \left\{\lambda>0: \int_{\mathbb{R}^{d}} \boldsymbol{v}_{\lambda}(x)(|h| *|\tilde{h}|)(x) \mathrm{d} x<\delta\right\} \quad \text { for all } \delta>0
$$

where $\inf \varnothing:=\infty$.

Theorem 5.3. Assume that $f=h * \tilde{h}$ with $h \in \mathcal{G}_{p}\left(\mathbb{R}^{d}\right)$ for some $p>1$. Then, the SPDE (1.1), subject to non-random initial data $u(0)=u_{0} \in L^{\infty}\left(\mathbb{R}^{d}\right)$ and non degeneracy condition $\operatorname{Lip}(\sigma)>0$, has a mild solution $u$ which is unique (up to a modification) subject to the additional condition (1.5) Finally, $(0, \infty) \times \mathbb{R}^{d} \ni(t, x) \mapsto u(t, x)$ is continuous in $L^{k}(\Omega)$ for very $k \geq 2$, and hence Lebesgue measurable (up to evanescence).

Outline of the proof of Theorem 5.3. The proof follows a standard route. We therefore outline it, in part to document the veracity of the argument, but mainly as a means of introducing objects that we will need later on.

Let $u_{0}(t, x):=u_{0}(x)$ for all $t \geq 0$ and $x \in \mathbb{R}^{d}$, and define iteratively

$$
\begin{aligned}
u_{n+1}(t, x): & =\int_{\mathbb{R}^{d}} \boldsymbol{p}_{t}(y-x) u_{0}(y) \mathrm{d} y+\int_{(0, t) \times \mathbb{R}^{d}} \boldsymbol{p}_{t-s}(x-y) \sigma\left(u_{n}(s, y)\right) \eta^{(h)}(\mathrm{d} s \mathrm{~d} y) \\
& =\left(\boldsymbol{p}_{t} * u_{0}\right)(t)+\left(\boldsymbol{p} \circledast \sigma\left(u_{n}\right) \eta^{(h)}\right)(t, x),
\end{aligned}
$$

for every integer $n \geq 0$ and all real numbers $t \geq 0$ and $x \in \mathbb{R}^{d}$. Then $u_{0}, u_{1}, \ldots$ represent the successive approximations of $u$ via Picard iteration. Since the first term is bounded uniformly by $\left\|u_{0}\right\|_{L^{\infty}\left(\mathbb{R}^{d}\right)}$, and since every $\mathcal{N}_{\beta, k}$ is a norm for every $\beta>0$ and $k \geq 1$, it follows that for all integers $n \geq 0$, and all reals $\beta>0$ and $k \geq 2$,

$$
\begin{aligned}
\mathcal{N}_{\beta, k}\left(u_{n+1}\right) & \leq\left\|u_{0}\right\|_{L^{\infty}\left(\mathbb{R}^{d}\right)}+\mathcal{N}_{\beta, k}\left(\boldsymbol{p} \circledast \sigma\left(u_{n}\right) \eta^{(h)}\right) \\
& \leq\left\|u_{0}\right\|_{L^{\infty}\left(\mathbb{R}^{d}\right)}+z_{k} \mathcal{N}_{\beta, k}\left(\sigma\left(u_{n}\right)\right) \sqrt{\frac{1}{2} \int_{\mathbb{R}^{d}} \boldsymbol{v}_{\beta}(x)|f(x)| \mathrm{d} x}
\end{aligned}
$$

see Lemma 5.2. Because $|\sigma(z)| \leq|\sigma(0)|+\operatorname{Lip}(\sigma)|z|$ for all $z \in \mathbb{R}$, it follows that

$$
\mathcal{N}_{\beta, k}\left(u_{n+1}\right) \leq\left\|u_{0}\right\|_{L^{\infty}\left(\mathbb{R}^{d}\right)}+z_{k}\left(|\sigma(0)|+\operatorname{Lip}(\sigma) \mathcal{N}_{\beta, k}\left(u_{n}\right)\right) \sqrt{\frac{1}{2} \int_{\mathbb{R}^{d}} \boldsymbol{v}_{\beta}(x)(|h| *|\tilde{h}|)(x) \mathrm{d} x}
$$

This is valid for every $\beta>0$ and $k \geq 2$.

Choose and fix $\varepsilon \in(0,1)$. Because

$$
\beta \geq \Lambda_{h}\left(\frac{2(1-\varepsilon)^{2}}{\left[z_{k} \operatorname{Lip}(\sigma)\right]^{2}}\right) \quad \text { iff } \quad \int_{\mathbb{R}^{d}} \boldsymbol{v}_{\beta}(x)(|h| *|\tilde{h}|)(x) \mathrm{d} x \leq \frac{2(1-\varepsilon)^{2}}{\left[z_{k} \operatorname{Lip}(\sigma)\right]^{2}}
$$


it follows that, under the condition $\beta \geq \Lambda_{h}\left(2(1-\varepsilon)^{2} /\left[z_{k} \operatorname{Lip}(\sigma)\right]^{2}\right)$,

$$
\begin{aligned}
\mathcal{N}_{\beta, k}\left(u_{n+1}\right) & \leq\left\|u_{0}\right\|_{L^{\infty}\left(\mathbb{R}^{d}\right)}+z_{k}|\sigma(0)| \sqrt{\frac{1}{2} \int_{\mathbb{R}^{d}} \boldsymbol{v}_{\beta}(x)(|h| *|\tilde{h}|) \mathrm{d} x}+(1-\varepsilon) \mathcal{N}_{\beta, k}\left(u_{n}\right) \\
& \leq\left\|u_{0}\right\|_{L^{\infty}\left(\mathbb{R}^{d}\right)}+\frac{|\sigma(0)|}{\operatorname{Lip}(\sigma)}+(1-\varepsilon) \mathcal{N}_{\beta, k}\left(u_{n}\right) \\
& \leq\left\|u_{0}\right\|_{L^{\infty}\left(\mathbb{R}^{d}\right)}+\frac{|\sigma(0)|}{\operatorname{Lip}(\sigma)}+(1-\varepsilon)\left[\left\|u_{0}\right\|_{L^{\infty}\left(\mathbb{R}^{d}\right)}+\frac{|\sigma(0)|}{\operatorname{Lip}(\sigma)}\right]+(1-\varepsilon)^{2} \mathcal{N}_{\beta, k}\left(u_{n-1}\right) \\
& \leq \cdots \leq\left[\left\|u_{0}\right\|_{L^{\infty}\left(\mathbb{R}^{d}\right)}+\frac{|\sigma(0)|}{\operatorname{Lip}(\sigma)}\right] \cdot\left[\sum_{j=0}^{n}(1-\varepsilon)^{j}+(1-\varepsilon)^{n+1}\left\|u_{0}\right\|_{L^{\infty}\left(\mathbb{R}^{d}\right)}\right] \\
& \leq\left[\left\|u_{0}\right\|_{L^{\infty}\left(\mathbb{R}^{d}\right)}+\frac{|\sigma(0)|}{\operatorname{Lip}(\sigma)}\right] \cdot\left[\frac{1}{\varepsilon}+(1-\varepsilon)^{n+1}\left\|u_{0}\right\|_{L^{\infty}\left(\mathbb{R}^{d}\right)}\right],
\end{aligned}
$$

after iteration. Similarly, one finds that

$$
\begin{aligned}
\mathcal{N}_{\beta, k}\left(u_{n+1}-u_{n}\right) & \leq \mathcal{N}_{\beta, k}\left(\boldsymbol{p} \circledast\left[\sigma\left(u_{n}\right)-\sigma\left(u_{n-1}\right)\right] \eta^{(h)}\right) \\
& \leq z_{k} \mathcal{N}_{\beta, k}\left(\sigma\left(u_{n}\right)-\sigma\left(u_{n-1}\right)\right) \sqrt{\frac{1}{2} \int_{\mathbb{R}^{d}} \boldsymbol{v}_{\beta}(x)(|h| *|\tilde{h}|) \mathrm{d} x} \\
& \leq z_{k} \operatorname{Lip}(\sigma) \mathcal{N}_{\beta, k}\left(u_{n}-u_{n-1}\right) \sqrt{\frac{1}{2} \int_{\mathbb{R}^{d}} \boldsymbol{v}_{\beta}(x)(|h| *|\tilde{h}|) \mathrm{d} x} \\
& \leq(1-\varepsilon) \mathcal{N}_{\beta, k}\left(u_{n}-u_{n-1}\right),
\end{aligned}
$$

provided still that $\beta \geq \Lambda_{h}\left(2(1-\varepsilon)^{2} /\left[z_{k} \operatorname{Lip}(\sigma)\right]^{2}\right)$. It follows immediately that $\left\{u_{n}\right\}_{n \geq 0}$ is a Cauchy sequence in $\mathbb{W}_{\beta, k}$ when $\beta \geq \Lambda_{h}\left(2(1-\varepsilon)^{2} /\left[z_{k} \operatorname{Lip}(\sigma)\right]^{2}\right)$. It also implies readily that $u:=\lim _{n \rightarrow \infty} u_{n}$ is an element of $\mathbb{W}_{\beta, k}$, for the same range of $\beta$ 's, and that $u$ solves (1.1). This and Fatou's lemma together prove the asserted upper bound for $\mathrm{E}\left(|u(t, x)|^{k}\right)$ as well.

The proof of uniqueness is also essentially standard: Suppose there existed $u, v \in$ $\mathrm{W}_{\beta, k}$ for some $\beta \geq \Lambda_{h}\left(2(1-\varepsilon)^{2} /\left[z_{k} \operatorname{Lip}(\sigma)\right]^{2}\right)$ both of which are mild solutions to (1.1). Then the same argument that led to (5.12) yields

$$
\mathcal{N}_{\beta, k, T}(u-v) \leq(1-\varepsilon) \mathcal{N}_{\beta, k, T}(u-v),
$$

for all $\beta \geq \Lambda_{h}\left(2(1-\varepsilon)^{2} /\left[z_{k} \operatorname{Lip}(\sigma)\right]^{2}\right)$ and $T>0$, where

$$
\mathcal{N}_{\beta, k, T}(\Phi):=\sup _{t \in[0, T]} \sup _{x \in \mathbb{R}^{d}} \mathrm{e}^{-\beta t}\|\Phi(t, x)\|_{k} ;
$$

compare with (5.1). In particular, it follows that there exists $\beta>0$ such that

$$
\mathcal{N}_{\beta, k, T}(u-v)=0 \quad \text { for all } T>0,
$$

and hence $u$ and $v$ are modifications of one another. We can unscramble the latter displayed statement in order to see that this yields the asserted bound for $\mathrm{E}\left(|u(t, x)|^{k}\right)$. Similarly, one proves $L^{k}(\Omega)$ continuity, which completes our (somewhat abbreviated) proof of Theorem 5.3.

Remark 5.4. Let us pause and record the following - see [19, eq. (54)] - ready byproduct of Theorem 5.3 and the Lipschitz continuity of $\sigma$ : For all $T>0$ and $k \geq 2$,

$$
C_{T, k}:=\sup _{n \geq 0} \sup _{(t, x) \in[0, T] \times \mathbb{R}^{d}} \mathrm{E}\left(\left|\sigma\left(u_{n}(t, x)\right)\right|^{k}\right)<\infty,
$$


where $u_{n}$ denotes the $n$ th-stage Picard iteration of the SPDE (1.1). Eq. (5.13) is valid also in the case that $f$ satisfies (1.8); see (5.11) for some $h \in \cup_{p>1} \mathcal{G}_{p}\left(\mathbb{R}^{d}\right)$.

Remark 5.5. Because of $L^{k}(\Omega)$-continuity, Doob's theory of separability becomes applicable (see Doob [22]) and implies, among other things, that $x \mapsto u(t, x)$ is Lebesgue measurable. This is of course directly relevant to the present discussion of spatial ergodicity.

\section{Malliavin calculus}

\subsection{A Clark-Ocone formula and a Poincaré inequality}

Suppose that the spatial correlation $f$ of the noise is a measure that satisfies Dalang's condition (1.4), or is a function of the form $f=h * \tilde{h}$ where $|h| \in H_{-1}\left(\mathbb{R}^{d}\right)$. Let $\mathcal{H}_{0}$ be the Hilbert space defined as the completion of $C_{c}^{\infty}\left(\mathbb{R}^{d}\right)$ under the scalar product

$$
\langle\phi, \varphi\rangle_{\mathcal{H}_{0}}=\langle\phi, \varphi * f\rangle_{L^{2}\left(\mathbb{R}^{d}\right)},
$$

and let $\mathcal{H}:=L^{2}\left(\mathbb{R}_{+} ; \mathcal{H}_{0}\right)$. Then the Gaussian family $\{\eta(\phi)\}_{\phi \in \mathcal{H}}$, described by the family of Walsh-type stochastic integrals,

$$
\eta(\phi)=\int_{\mathbb{R}_{+} \times \mathbb{R}^{d}} \phi(s, x) \eta(\mathrm{d} s \mathrm{~d} x),
$$

defines an isonormal Gaussian process on the Hilbert space $\mathcal{H}$. When $f=h * \tilde{h}$, we can use the noise $\eta^{(h)}$ to construct this integral from the integral with respect to a space-time white noise as it has been done in $§ 5.1$.

In this framework, we can develop the Malliavin calculus with respect to the noise $\eta$. Next we recall some of the basic definitions of that Malliavin calculus.

Denote by $\mathcal{S}$ be the set of smooth and cylindrical random variables of the form

$$
F=\Psi\left(\eta\left(\phi_{1}\right), \ldots, \eta\left(\phi_{n}\right)\right),
$$

where $\Psi \in C_{c}^{\infty}\left(\mathbb{R}^{n}\right)$ and $\phi=\left(\phi_{1}, \ldots, \phi_{n}\right) \in \mathcal{H}^{n}$. If $F \in \mathcal{S}$ has the above form, then the Malliavin derivative $D F$ is the $\mathcal{H}$-valued random variable defined by

$$
D F:=(\nabla \Psi)\left(\eta\left(\phi_{1}\right), \ldots, \eta\left(\phi_{n}\right)\right) \cdot \phi=\sum_{i=1}^{n}\left(\partial_{i} \Psi\right)\left(\eta\left(\phi_{1}\right) \ldots, \eta\left(\phi_{n}\right)\right) \phi_{i} .
$$

In particular, $D(\eta(\varphi))=\varphi$ or every $\varphi \in \mathcal{H}$; that is, $D$ can be interpreted as the inverse of the Wiener stochastic-integral operator $\phi \mapsto \eta(\phi)$.

The operator $D$ is a closable linear mapping from $L^{p}(\Omega)$ to $L^{p}(\Omega ; \mathcal{H})$ for every real number $p \geq 1$. We can define the Gaussian Sobolev space $\mathbb{D}^{1, p}$ as the closure of $\mathcal{S}$ with respect to the seminorm $\|\cdot\|_{1, p}$, defined via

$$
\|F\|_{1, p}^{p}:=\mathrm{E}\left(|F|^{p}\right)+\mathrm{E}\left(\|D F\|_{\mathcal{H}}^{p}\right) .
$$

We will make use of the notation $D_{s, z} F$ to represent the derivative as a random field, indexed by $(s, z) \in \mathbb{R}_{+} \times \mathbb{R}^{d}$. In particular, if $F=u(t, x)$, then $D_{r, z} u(t, x)$ will serve as short-hand for $D_{r, z}[u(t, x)]$.

The divergence operator $\delta$ is defined as the adjoint of $D$. More precisely, we first define the domain of $\delta$ - denoted by Dom $\delta$ - as the set of random elements $v \in L^{2}(\Omega ; \mathcal{H})$ for which we can find a real number $c_{v}>0$ such that

$$
\left|\mathrm{E}\left(\langle v, D F\rangle_{\mathcal{H}}\right)\right| \leq c_{v}\|F\|_{L^{2}(\Omega)} \quad \text { for every } F \in \mathbb{D}^{1,2} \text {. }
$$


For every $v \in \operatorname{Dom} \delta$, we define the real-valued random variable $\delta(v)$ via the following duality relation:

$$
\mathrm{E}\left[\langle D F, v\rangle_{\mathcal{H}}\right]=\mathrm{E}[F \delta(v)] \quad \text { for every } F \in \mathbb{D}^{1,2} .
$$

It turns out that $\delta$ is a closed operator. This means that, if $v_{1}, v_{2}, \ldots \in$ Dom $\delta$ satisfy $\lim _{n \rightarrow \infty} v_{n}=v$ in $L^{2}(\Omega ; \mathcal{H})$, and if $G:=\lim _{n \rightarrow \infty} \delta\left(v_{n}\right)$ exists in $L^{2}(\Omega)$, then $v \in \operatorname{Dom} \delta$, and $\delta(v)=G$. $\delta$.

Next, we provide two examples of elements of the domain of the divergence operator

Example 6.1. Suppose that $v \in L^{2}\left(\Omega \times \mathbb{R}_{+} ; \mathcal{H}_{0}\right)$ is a smooth and cylindrical $\mathcal{H}_{0}$-valued stochastic process of the form $v(t)=\sum_{j=1}^{n} F_{j} \phi_{j}(t)$ where the $F_{j} \in \mathcal{S}$, and $\phi_{j} \in \mathcal{H}_{0}$ for all $j=1, \ldots, n$. Then, $v \in \operatorname{Dom} \delta$, and

$$
\delta(v)=\sum_{j=1}^{n} F_{j} \eta\left(\phi_{j}\right)-\sum_{j=1}^{n}\left\langle D F_{j}, \phi_{j}\right\rangle_{\mathcal{H}} .
$$

This property follows immediately from (6.1).

Example 6.2. Consider a predictable random field $\{H(s, y)\}_{s \geq 0, y \in \mathbb{R}^{d}}$ such that the Walsh integral $\int_{\mathbb{R}_{+} \times \mathbb{R}^{d}} H \mathrm{~d} \eta$ is well defined and in $L^{2}(\Omega)$. Then, $H \in \operatorname{Dom} \delta$ as an $\mathcal{H}_{0^{-}}$ valued process, and $\delta(H)$ coincides with the Walsh stochastic integral of $H$; that is, $\delta(H)=\int_{\mathbb{R}_{+} \times \mathbb{R}^{d}} H \mathrm{~d} \eta$. This result is well-known in the case of stochastic integrals with respect to the Brownian motion (see [27] and also [46, Proposition 1.3.11]). The same proof works for $\mathcal{H}_{0}$-valued processes.

The Clark-Ocone formula will play a fundamental role in the proof of our results. We state below this formula and give a proof for the sake of completeness. Throughout, we denote by $\mathcal{F}:=\left\{\mathcal{F}_{t}\right\}_{t \geq 0}$ the natural filtration of the noise $\eta$; that is, $\mathcal{F}$ is the usual augmentation of the filtration $\mathcal{F}^{0}$, defined via $\mathcal{F}_{0}^{0}:=\{\varnothing, \Omega\}$ and

$$
\mathcal{F}_{t}^{0}:=\text { sigma-algebra generated by }\left\{\int_{(0, r) \times \mathbb{R}^{d}} \phi(x) \eta(\mathrm{d} s \mathrm{~d} x) ; 0 \leq r \leq t\right\} \quad \text { for all } t>0,
$$

as $\phi$ ranges over all elements of $C_{c}\left(\mathbb{R}^{d}\right)$; see also (1.2).

Proposition 6.3 (A Clark-Ocone formula/Poincaré inequality). For every $F \in \mathbb{D}^{1,2}$,

$$
F=\mathrm{E} F+\int_{\mathbb{R}_{+} \times \mathbb{R}^{d}} \mathrm{E}\left(D_{s, z} F \mid \mathcal{F}_{s}\right) \eta(\mathrm{d} s \mathrm{~d} z) \quad \text { a.s. }
$$

Consequently, we have the Poincaré inequality, $\operatorname{Var}(F) \leq \mathrm{E}\left(\|D F\|_{\mathcal{H}}^{2}\right)$.

Proof. It suffices to prove the integral representation of $F$; the Poincaré inequality follows from the integral representation by the same argument as in (1.15), using the spatial covariance structure of $\eta$; see (1.2).

One can extend the martingale representation theorem, proved by Doob in [22], to martingales that take value in a Hilbert space [41, 48]. It follows from that extension that there exists a unique $\mathcal{H}_{0}$-valued predictable process $H$ such that the Walsh integral $\int_{\mathbb{R}_{+} \times \mathbb{R}^{d}} H \mathrm{~d} \eta$ is well-defined in $L^{2}(\Omega)$, and

$$
F=\mathrm{E} F+\int_{\mathbb{R}_{+} \times \mathbb{R}^{d}} H(s, z) \eta(\mathrm{d} s \mathrm{~d} z) .
$$

It remains to prove that

$$
H(s, z)=\mathrm{E}\left(D_{s, z} F \mid \mathcal{F}_{s}\right),
$$


viewed as in identity in $L^{2}\left(\Omega \times \mathbb{R}_{+} ; \mathcal{H}_{0}\right)$. We will prove (6.3) in the case that $f$ is a nonnegative-definite function. The more general case where $f$ is a nonnegative distribution follows in exactly the same way, but one has to adjust the ensuing integrals for example so that $\int \psi(x) f(x) \mathrm{d} x$ is replaced by $f(\psi)$, etc. Proving the more general case requires no new ideas, only the introduction of heavy-handed notation. Therefore, we stick to the less notation-intensive case that $f$ is a function.

Both sides of (6.3) define predictable random fields. Therefore, it suffices to prove that

$$
\begin{aligned}
\int_{0}^{\infty} \mathrm{d} s \int_{\mathbb{R}^{d}} \mathrm{~d} y \int_{\mathbb{R}^{d}} \mathrm{~d} z \mathrm{E}[H(s, y) v(s, z)] f(y-z) \\
\quad=\int_{0}^{\infty} \mathrm{d} s \int_{\mathbb{R}^{d}} \mathrm{~d} y \int_{\mathbb{R}^{d}} \mathrm{~d} z \mathrm{E}\left[\mathrm{E}\left(D_{s, y} F \mid \mathcal{F}_{s}\right) v(s, z)\right] f(y-z),
\end{aligned}
$$

for every predictable process $v \in L^{2}\left(\Omega \times \mathbb{R}_{+} ; \mathcal{H}_{0}\right)$. Since $v(s, \cdot)$ is $\mathcal{F}_{s}$-measurable for every $s>0$, the duality relation (6.1) implies that

$$
\begin{aligned}
\int_{0}^{\infty} \mathrm{d} s \int_{\mathbb{R}^{d}} \mathrm{~d} y \int_{\mathbb{R}^{d}} \mathrm{~d} z \mathrm{E}\left[\mathrm{E}\left(D_{s, y} F \mid \mathcal{F}_{s}\right) \cdot v(s, z)\right] f(y-z) \\
\quad=\int_{0}^{\infty} \mathrm{d} s \int_{\mathbb{R}^{d}} \mathrm{~d} y \int_{\mathbb{R}^{d}} \mathrm{~d} z \mathrm{E}\left[D_{s, y} F \cdot v(s, z)\right] f(y-z)=\mathrm{E}\left[\langle D F, v\rangle_{\mathcal{H}}\right]=\mathrm{E}[F \delta(v)] .
\end{aligned}
$$

Because $\delta(v)$ coincides with the Walsh integral of $v$, (6.2) implies that

$\mathrm{E}[F \delta(v)]=\mathrm{E}\left[\int_{\mathbb{R}_{+} \times \mathbb{R}^{d}} H \mathrm{~d} \eta \cdot \int_{\mathbb{R}_{+} \times \mathbb{R}^{d}} v \mathrm{~d} \eta\right]=\int_{0}^{\infty} \mathrm{d} s \int_{\mathbb{R}^{d}} \mathrm{~d} y \int_{\mathbb{R}^{d}} \mathrm{~d} z \mathrm{E}[H(s, y) v(s, z)] f(y-z)$,

thanks to the $L^{2}(\Omega)$-isometry of the Walsh stochastic integral. This concludes the proof.

\subsection{Differentiability of the solution}

In order to apply Malliavin calculus, we first need to check that the solution to (1.1) is differentiable in the sense of Malliavin calculus. This section is concerned with that, which we state next in the following comprehensive form.

Theorem 6.4. Suppose $f$ satisfies either Dalang's condition (1.4), or condition (1.8) with $h \in \cup_{p>1} \mathcal{G}_{p}\left(\mathbb{R}^{d}\right)$, and let $u$ denote the mild solution to (1.1). Then,

$$
u(t, x) \in \bigcap_{k \geq 2} \mathbb{D}^{1, k} \quad \text { for every }(t, x) \in(0, \infty) \times \mathbb{R}^{d} .
$$

Moreover, if $t \in(0, T)$ for a fixed $T>0$ and $x \in \mathbb{R}^{d}$, then

$$
\left\|D_{s, y} u(t, x)\right\|_{k} \leq \frac{\sqrt{2} C_{T, k} \mathrm{e}^{\lambda_{0}(t-s)}}{\sqrt{1-2^{(d+2) / 2}\left[z_{k} \operatorname{Lip}(\sigma)\right]^{2}\left(\boldsymbol{v}_{\lambda_{0}} * \overline{\mathbf{f}}\right)(0)}} \boldsymbol{p}_{t-s}(x-y),
$$

for almost every $(s, y) \in(0, t) \times \mathbb{R}^{d}$. The quantities $C_{T, k}, z_{k}$, and $\boldsymbol{v}_{\lambda_{0}}$ are respectively defined in (5.13), (5.6), and (3.8), $\lambda_{0}>0$ is arbitrary but large enough to ensure that

$$
\left(\boldsymbol{v}_{\lambda_{0}} * \overline{\mathbf{f}}\right)(0)<\frac{1}{2^{(d+2) / 2}\left[z_{k} \operatorname{Lip}(\sigma)\right]^{2}},
$$

and

$$
\overline{\mathbf{f}}:= \begin{cases}f & \text { when } f \text { satisfies (1.4), } \\ |h| *|\tilde{h}| & \text { when } f \text { satisfies (1.8) for some } h \in \bigcup_{p>1} \mathcal{G}_{p}\left(\mathbb{R}^{d}\right) .\end{cases}
$$


Remark 6.5. In the case that $f$ is a nonnegative function that satisfies Dalang's condition (1.4), the first part of this theorem - namely that $u(t, x) \in \cap_{k \geq 2} \mathbb{D}^{1, k}$ - was proved by Chen and Huang [11, Proposition 3.2].

The proof of Theorem 6.4 first requires some preliminary development, which we present as two lemmas.

Lemma 6.6. Choose and fix a real number $T>0$. Then, for every nondecreasing function $g:[0, T] \mapsto \mathbb{R}_{+}$and for all $t \in(0, T)$ and $y \in \mathbb{R}^{d}$,

$$
\int_{0}^{t} g(s) \boldsymbol{p}_{2 s(t-s) / t}(y) \mathrm{d} s \leq 2^{(d+2) / 2} \int_{0}^{t} g(s) \boldsymbol{p}_{2(t-s)}(y) \mathrm{d} s .
$$

Proof. The proof is similar to Lemma B.1 of Chen and Huang [10]. Since $g$ is monotone,

$$
\int_{0}^{t / 2} g(s) \boldsymbol{p}_{2 s(t-s) / t}(y) \mathrm{d} s=\int_{t / 2}^{t} g(t-s) \boldsymbol{p}_{2 s(t-s) / t}(y) \mathrm{d} s \leq \int_{t / 2}^{t} g(s) \boldsymbol{p}_{2 s(t-s) / t}(y) \mathrm{d} s .
$$

Hence,

$$
\begin{aligned}
\int_{0}^{t} g(s) \boldsymbol{p}_{2 s(t-s) / t}(y) \mathrm{d} s & \leq 2 \int_{t / 2}^{t} g(s) \boldsymbol{p}_{2 s(t-s) / t}(y) \mathrm{d} s \\
& =2 \int_{t / 2}^{t} g(s) \exp \left(-\frac{t\|y\|^{2}}{4 s(t-s)}\right) \frac{\mathrm{d} s}{(4 \pi s(t-s) / t)^{d / 2}} \\
& \leq 2^{(d+2) / 2} \int_{t / 2}^{t} g(s) \exp \left(-\frac{\|y\|^{2}}{4(t-s)}\right) \frac{\mathrm{d} s}{(4 \pi(t-s))^{d / 2}} \\
& =2^{(d+2) / 2} \int_{t / 2}^{t} g(s) \boldsymbol{p}_{2(t-s)}(y) \mathrm{d} s,
\end{aligned}
$$

which clearly is bounded from above by $2^{(d+2) / 2} \int_{0}^{t} g(s) \boldsymbol{p}_{2(t-s)} \mathrm{d} s$.

In order to estimate the $L^{k}$-norm of the Malliavin derivative of the solution, we introduce some notations which will be used later on.

$$
\kappa(t):=\left(\boldsymbol{p}_{2 t} * \overline{\mathbf{f}}\right)(0),
$$

for the same distribution $\overline{\mathrm{f}}$ that was defined in the statement of Theorem 6.4. Next, define $h_{0}(t) \equiv 1$ and

$$
h_{n}(t):=\int_{0}^{t} h_{n-1}(s) \kappa(t-s) \mathrm{d} s \quad \text { for all } t>0 \text { and } n \geq 1 .
$$

By induction, it is clear that the function $h_{n}$ is nondecreasing for all $n \geq 0$. [The functions $\left\{h_{n}\right\}_{n \geq 0}$ should not be confused with the function $h$ in (1.8).]

We now follow Chen and Huang [10] and define for every $\gamma \geq 0$ and $t>0$,

$$
H(t ; \gamma):=\sum_{n=0}^{\infty} \gamma^{n} h_{n}(t)
$$

Recall the $\lambda$-potential density of the heat semigroup on $\mathbb{R}^{d}$ defined in (3.8).

Lemma 6.7. Suppose $f$ satisfies either (1.4), or (1.8) with $h \in \cup_{p>1} \mathcal{G}_{p}\left(\mathbb{R}^{d}\right)$. Then, for all $\gamma \geq 0$ and $t \geq 0$, the following inequality holds

$$
H(t ; \gamma) \leq \frac{\mathrm{e}^{2 \lambda t}}{1-\frac{1}{2} \gamma\left(\boldsymbol{v}_{\lambda} * \overline{\mathbf{f}}\right)(0)} \quad \text { for all } t>0,
$$

provided that $\lambda>0$ and $0 \leq \gamma<2 /\left(\boldsymbol{v}_{\lambda} * \overline{\mathbf{f}}\right)(0)$. 
Proof. Define $\mu_{n}:=\sup _{t>0}\left[\mathrm{e}^{-2 \lambda t} h_{n}(t)\right]$ for every integer $n \geq 0$, and note that $h_{n+1}(t)=\int_{0}^{t} h_{n}(s) \kappa(t-s) \mathrm{d} s \leq \mathrm{e}^{2 \lambda t} \mu_{n} \int_{0}^{t} \mathrm{e}^{-2 \lambda(t-s)} \kappa(t-s) \mathrm{d} s \leq \mathrm{e}^{2 \lambda t} \mu_{n} \int_{0}^{\infty} \mathrm{e}^{-2 \lambda s} \kappa(s) \mathrm{d} s$, for all $n \geq 0$ and $t>0$. Thus, $\mu_{n+1} \leq \frac{1}{2} \mu_{n}\left(\boldsymbol{v}_{\lambda} * \overline{\mathbf{f}}\right)(0)$ for all $n \geq 0$. Since $\mu_{0}=1$, we are led to

$$
h_{n}(t) \leq\left[\frac{1}{2}\left(\boldsymbol{v}_{\lambda} * \overline{\mathbf{f}}\right)(0)\right]^{n} \quad \text { for all } t>0 \text { and } n \geq 0,
$$

which leads to the lemma.

We are now ready to prove Theorem 6.4.

Proof of Theorem 6.4. Throughout, we choose and fix a real number $T>0$. We will prove the result in the case that $f$ is a function that satisfies either (1.4), or (1.8) for some $h \in \cup_{p>1} \mathcal{G}_{p}\left(\mathbb{R}^{d}\right)$. The remaining case is when $f$ is a measure that satisfies Dalang's condition (1.4); that case is proved by making only small adjustments to the following argument, but requires the introduction of a good deal of notation. Therefore, we consider only the case that $f$ is a function. Note in particular, that $\overline{\mathbf{f}}$ is also a function, and regardless of whether or not $f$ is signed, we always have $|f| \leq|\overline{\mathbf{f}}|$. From here on, we adapt the iterative method of [10, Lemma 2.2].

Let $u_{0}(t, x):=1$ for all $t>0$ and $x \in \mathbb{R}^{d}$, and recall the Picard iterations introduced in (5.8):

$$
u_{n+1}(t, x):=1+\int_{\mathbb{R}_{+} \times \mathbb{R}^{d}} \boldsymbol{p}_{t-s}(x-y) \sigma\left(u_{n}(s, y)\right) \eta(\mathrm{d} s \mathrm{~d} y),
$$

for all $n \geq 0, t>0$, and $x \in \mathbb{R}^{d}$.

Let $C_{T, k}$ be the constant in (5.13) and define

$$
\gamma:=2^{(d+4) / 2}\left[z_{k} \operatorname{Lip}(\sigma)\right]^{2},
$$

where $z_{k}$ was defined in (5.6). We claim that, for the above choice of $\gamma, u_{n}(t, x) \in \mathbb{D}^{1, k}$ for every $(t, x) \in(0, T) \times \mathbb{R}^{d}$ and $k \geq 2$, and

$$
\left\|D_{s, y} u_{n}(t, x)\right\|_{k} \leq \sqrt{2} C_{T, k} \boldsymbol{p}_{t-s}(x-y)\left(\sum_{i=0}^{n} \gamma^{i} h_{i}(t-s)\right)^{1 / 2},
$$

for almost every $(s, y) \in(0, t) \times \mathbb{R}^{d}$, where the functions $h_{i}$ are defined in (6.7). Let $\left(P_{n}\right)$ denote this logical proposition. Clearly $\left(P_{0}\right)$ holds, as the left-hand side of (6.9) is equal to zero. Now suppose $\left(P_{k}\right)$ holds for every integer $k=0, \ldots, n$, where $n \geq 0$ is integer. We propose to derive the conditional truth of $\left(P_{n+1}\right)$. This will be enough to prove (6.9) inductively.

According to Proposition 1.2.4 of Nualart [46], $\sigma\left(u_{n}(t, x)\right) \in \mathbb{D}^{1, k}$ for every $(t, x) \in$ $(0, T) \times \mathbb{R}^{d}$; moreover,

$$
D\left(\sigma\left(u_{n}(t, x)\right)\right)=\Sigma_{n} D u_{n}(t, x) \quad \text { a.s. }
$$

where $\Sigma_{n}:=\sigma^{\prime}\left(u_{n}(t, x)\right)$ for any version of the derivative $\sigma^{\prime}$. This is because, on the event $\left\{\left\|D u_{n}(t, x)\right\|_{\mathcal{H}}>0\right\}$, the random variable $u_{n}(t, x)$ is absolutely continuous.

We apply the properties of the divergence operator (see [46, Prop. 1.3.8]) in order to find that $\int_{(0, t) \times \mathbb{R}^{d}} \boldsymbol{p}_{t-s}(x-y) \sigma\left(u_{n}(s, y)\right) \eta(\mathrm{d} s \mathrm{~d} y) \in \mathbb{D}^{1, k}$. Moreover,

$$
\begin{aligned}
D_{r, z} u_{n+1}(t, x)= & D_{r, z}\left(\int_{(0, t) \times \mathbb{R}^{d}} \boldsymbol{p}_{t-s}(x-y) \sigma\left(u_{n}(s, y)\right) \eta(\mathrm{d} s \mathrm{~d} y)\right) \\
= & \boldsymbol{p}_{t-r}(x-z) \sigma\left(u_{n}(r, z)\right) \\
& +\int_{(r, t) \times \mathbb{R}^{d}} \boldsymbol{p}_{t-s}(x-y) \Sigma_{n} D_{r, z} u_{n}(s, y) \eta(\mathrm{d} s \mathrm{~d} y) \quad \text { a.s., }
\end{aligned}
$$


whence

$$
\begin{aligned}
\left\|D_{r, z} u_{n+1}(t, x)\right\|_{k} \leq \boldsymbol{p}_{t-r}(x-z)\left\|\sigma\left(u_{n}(r, z)\right)\right\|_{k} & \\
& +\left\|\int_{(r, t) \times \mathbb{R}^{d}} \boldsymbol{p}_{t-s}(x-y) \Sigma_{n} D_{r, z} u_{n}(s, y) \eta(\mathrm{d} s \mathrm{~d} y)\right\|_{k},
\end{aligned}
$$

for every integer $k \geq 2$. Define

$$
\mathcal{P}_{\tau}(y, w ; x):=\boldsymbol{p}_{\tau}(x-y) \boldsymbol{p}_{\tau}(x-w) \quad \text { for every } \tau>0 \text { and } x, y, w \in \mathbb{R}^{d} \text {. }
$$

Then the Burkholder-Davis-Gundy inequality [2, 3, 4] implies that

$$
\begin{aligned}
& \mathrm{E}\left(\left|\int_{(r, t) \times \mathbb{R}^{d}} \boldsymbol{p}_{t-s}(x-y) \Sigma_{n} D_{r, z} u_{n}(s, y) \eta(\mathrm{d} s \mathrm{~d} y)\right|^{k}\right) \\
& \leq\left[z_{k} \operatorname{Lip}(\sigma)\right]^{k} \mathrm{E}\left(\left|\int_{r}^{t} \mathrm{~d} s \int_{\mathbb{R}^{d}} \mathrm{~d} y \int_{\mathbb{R}^{d}} d w \mathcal{P}_{t-s}(y, w ; x)\right| D_{r, z} u_{n}(s, y)|| D_{r, z} u_{n}(s, w)|\overline{\mathbf{f}}(y-w)|^{k / 2}\right) .
\end{aligned}
$$

Back-to-back appeals to the inequalities of Minkowski and Cauchy-Schwarz then leads us to the following:

$$
\begin{aligned}
& \mathrm{E}\left(\left|\int_{(r, t) \times \mathbb{R}^{d}} \boldsymbol{p}_{t-s}(x-y) \Sigma_{n} D_{r, z} u_{n}(s, y) \eta(\mathrm{d} s \mathrm{~d} y)\right|^{k}\right) \\
& \leq\left[z_{k} \operatorname{Lip}(\sigma)\right]^{k}\left[\int_{r}^{t} \mathrm{~d} s \int_{\mathbb{R}^{d}} \mathrm{~d} y \int_{\mathbb{R}^{d}} \mathrm{~d} w \mathcal{P}_{t-s}(y, w ; x)\left\|D_{r, z} u_{n}(s, y) D_{r, z} u_{n}(s, w)\right\|_{k / 2} \overline{\mathbf{f}}(y-w)\right]^{k / 2} \\
& \leq\left[z_{k} \operatorname{Lip}(\sigma)\right]^{k}\left[\int_{r}^{t} \mathrm{~d} s \int_{\mathbb{R}^{d}} \mathrm{~d} y \int_{\mathbb{R}^{d}} \mathrm{~d} w \mathcal{P}_{t-s}(y, w ; x)\left\|D_{r, z} u_{n}(s, y)\right\|_{k}\left\|D_{r, z} u_{n}(s, w)\right\|_{k} \overline{\mathbf{f}}(y-w)\right]^{k / 2} .
\end{aligned}
$$

The preceding displayed computations yield the following inequality on the Malliavin derivative of $u_{n+1}(t, x)$ :

$$
\begin{aligned}
& \left\|D_{r, z} u_{n+1}(t, x)\right\|_{k} \\
& \leq C_{T, k} \boldsymbol{p}_{t-r}(x-z) \\
& +z_{k} \operatorname{Lip}(\sigma)\left[\int_{r}^{t} \mathrm{~d} s \int_{\mathbb{R}^{d}} \mathrm{~d} y \int_{\mathbb{R}^{d}} \mathrm{~d} w \mathcal{P}_{t-s}(y, w ; x)\left\|D_{r, z} u_{n}(s, y)\right\|_{k}\left\|D_{r, z} u_{n}(s, w)\right\|_{k} \overline{\mathbf{f}}(y-w)\right]^{1 / 2} .
\end{aligned}
$$

By our induction hypothesis, $\left(P_{n}\right)$ is valid; that is, $(6.9)$ holds [for $n$ ], whence

$$
\begin{aligned}
& \int_{r}^{t} \mathrm{~d} s \int_{\mathbb{R}^{d}} \mathrm{~d} y \int_{\mathbb{R}^{d}} \mathrm{~d} w \mathcal{P}_{t-s}(y, w ; x)\left\|D_{r, z} u_{n}(s, y)\right\|_{k}\left\|D_{r, z} u_{n}(s, w)\right\|_{k} \overline{\mathbf{f}}(y-w) \\
& \leq 2 C_{T, k}^{2} \sum_{i=0}^{n} \gamma^{i} \int_{r}^{t} \mathrm{~d} s h_{i}(s-r) \int_{\mathbb{R}^{d}} \mathrm{~d} y \int_{\mathbb{R}^{d}} \mathrm{~d} w \mathcal{P}_{t-s}(y, w ; x) \mathcal{P}_{s-r}(y, w ; z) \overline{\mathbf{f}}(y-w) .
\end{aligned}
$$

A careful inspection of the expression for $\mathcal{P}$ with elementary pointwise inequality ${ }^{5}$

$$
\boldsymbol{p}_{t-s}(x-y) \boldsymbol{p}_{s}(y-z)=\boldsymbol{p}_{t}(x-z) \boldsymbol{p}_{s(t-s) / t}\left(y-z-\frac{s}{t}(x-z)\right)
$$

\footnotetext{
${ }^{5}$ See for example the formula below (2.10) in Chen and Huang [10].
} 
yields the following upper bound for the quantity on the right-hand side of (6.10):

$$
\begin{aligned}
& 2 C_{T, k}^{2} \boldsymbol{p}_{t-r}^{2}(x-z) \sum_{i=0}^{n} \gamma^{i} \int_{r}^{t} \mathrm{~d} s h_{i}(s-r) \int_{\mathbb{R}^{d}} \mathrm{~d} y \int_{\mathbb{R}^{d}} \mathrm{~d} w \overline{\mathbf{f}}(y-w) \\
& \quad \times \boldsymbol{p}_{(s-r)(t-s) /(t-r)}\left(y-z-\frac{s-r}{t-r}(x-z)\right) \boldsymbol{p}_{(s-r)(t-s) /(t-r)}\left(w-z-\frac{s-r}{t-r}(x-z)\right) \\
& =2 C_{T, k}^{2} \boldsymbol{p}_{t-r}^{2}(x-z) \sum_{i=0}^{n} \gamma^{i} \int_{0}^{t-r} \mathrm{~d} s h_{i}(s) \int_{\mathbb{R}^{d}} \mathrm{~d} y \overline{\mathbf{f}}(y) \boldsymbol{p}_{2 s(t-r-s) /(t-r)}(y),
\end{aligned}
$$

where the final identity can be deduced from a change of variables $[y-w \rightarrow y]$ and the semigroup property of the heat kernel.

Since every function $h_{i}$ is nondecreasing and $(a+b)^{2} \leq 2 a^{2}+2 b^{2}$ for all $a, b \geq 0$, Lemma 6.6 implies that

$$
\begin{aligned}
&\left\|D_{r, z} u_{n+1}(t, x)\right\|_{k}^{2} \leq 2 C_{T, k}^{2} \boldsymbol{p}_{t-r}^{2}(x-z)+2^{(d+6) / 2}\left[z_{k} \operatorname{Lip}(\sigma)\right]^{2} C_{T, k}^{2} \boldsymbol{p}_{t-r}^{2}(x-z) \\
& \quad \times \sum_{i=0}^{n} \gamma^{i} \int_{\mathbb{R}^{d}} \mathrm{~d} y \overline{\mathbf{f}}(y) \int_{0}^{t-r} \mathrm{~d} s h_{i}(s) \boldsymbol{p}_{2(t-r-s)}(y) \\
&=2 C_{T, k}^{2} \boldsymbol{p}_{t-r}^{2}(x-z) \\
&+2^{(d+6) / 2}\left[z_{k} \operatorname{Lip}(\sigma)\right]^{2} C_{T, k}^{2} \boldsymbol{p}_{t-r}^{2}(x-z) \sum_{i=0}^{n} \gamma^{i} h_{i+1}(t-r) \\
&= 2 C_{T, k}^{2} \boldsymbol{p}_{t-r}^{2}(x-z)\left(1+\gamma \sum_{i=0}^{n} \gamma^{i} h_{i+1}(t-r)\right) .
\end{aligned}
$$

This proves the conditional validity of the proposition $\left(P_{n+1}\right)$, given that $\left(P_{j}\right)$ is valid for all $j=0, \ldots, n$. Induction yields (6.9); we can now conclude the proof as follows.

Because $\lim _{n \rightarrow \infty} u_{n}(t, x)=u(t, x)$ in $L^{k}(\Omega)$, (6.9) and Lemma 6.7 together imply that

$$
\sup _{n \geq 0} \mathrm{E}\left(\left\|D u_{n}(t, x)\right\|_{\mathcal{H}}^{k}\right)<\infty .
$$

Lemma 1.5.3 of Nualart [46] now implies that $u(t, x) \in \mathbb{D}^{1, k}$.

Finally, it remains to show that the estimate (6.4) holds for $u(t, x)$, where $t \in(0, T)$ and $x \in \mathbb{R}^{d}$ are held fixed. This follows from the fact that $D u_{n}(t, x)$ converges in the weak topology of $L^{k}(\Omega ; \mathcal{H})$ to $D u(t, x)$ possibly after moving to a subsequence. This proof is a little bit involved and carried out as follows: First note that, because of (6.9),

$$
\sup _{n \geq 0} \mathrm{E}\left(\left\|D u_{n}(t, x)\right\|_{L^{k}\left(\mathbb{R}_{+} \times \mathbb{R}^{d}\right)}^{k}\right)<\infty \quad \text { for } 1 \leq k<\frac{2}{d}+1 .
$$

Fix such a $k$. It follows that, after possibly moving to subsequence, $D u_{n}(t, x)$ converges to $D u(t, x)$ in the weak topology of $L^{k}\left(\Omega ; \mathbb{R}_{+} \times \mathbb{R}^{d}\right)$, whence

$$
D u(t, x) \in L^{k}\left(\Omega ; \mathbb{R}_{+} \times \mathbb{R}^{d}\right) .
$$

Then, we use a smooth approximation $\left\{\psi_{\epsilon}\right\}_{\epsilon>0}$ to the identity in $\mathbb{R}_{+} \times \mathbb{R}^{d}$, and apply Fatou's lemma and duality for $L^{p}$-spaces in order to find that, for almost every $(s, y) \in$ $[0, t] \times \mathbb{R}^{d}$ and for all $k \geq 2$,

$$
\begin{aligned}
\left\|D_{s, y} u(t, x)\right\|_{k} & \leq \limsup _{\epsilon \rightarrow 0}\left\|\int_{\mathbb{R}_{+} \times \mathbb{R}^{d}} D_{s^{\prime}, y^{\prime}} u(t, x) \psi_{\epsilon}\left(s^{\prime}-s, y^{\prime}-y\right) \mathrm{d} s^{\prime} \mathrm{d} y^{\prime}\right\|_{k} \\
& \leq \limsup _{\epsilon \rightarrow 0} \sup _{\|G\|_{k /(k-1)} \leq 1}\left|\int_{\mathbb{R}_{+} \times \mathbb{R}^{d}} \mathrm{E}\left[G D_{s^{\prime}, y^{\prime}} u(t, x)\right] \psi_{\epsilon}\left(s^{\prime}-s, y^{\prime}-y\right) \mathrm{d} s^{\prime} \mathrm{d} y^{\prime}\right| .
\end{aligned}
$$


Choose and fix a random variable $G \in L^{k /(k-1)}(\Omega)$ such that $\mathrm{E}\left(|G|^{k /(k-1)}\right) \leq 1$. By (6.9), we can find a subsequence $n(1)<n(2)<\cdots$ of positive integers such that

$$
\begin{aligned}
& \left|\int_{\mathbb{R}_{+} \times \mathbb{R}^{d}} \mathrm{E}\left[G D_{s^{\prime}, y^{\prime}} u(t, x)\right] \psi_{\epsilon}\left(s^{\prime}-s, y^{\prime}-y\right) \mathrm{d} s^{\prime} \mathrm{d} y^{\prime}\right| \\
& =\lim _{\ell \rightarrow \infty}\left|\int_{\mathbb{R}_{+} \times \mathbb{R}^{d}} \mathrm{E}\left[G D_{s^{\prime}, y^{\prime}} u_{n(\ell)}(t, x)\right] \psi_{\epsilon}\left(s^{\prime}-s, y^{\prime}-y\right) \mathrm{d} s^{\prime} \mathrm{d} y^{\prime}\right| \\
& \leq \limsup _{\ell \rightarrow \infty} \int_{\mathbb{R}_{+} \times \mathbb{R}^{d}}\left\|D_{s^{\prime}, y^{\prime}} u_{n(\ell)}(t, x)\right\|_{k} \psi_{\epsilon}\left(s^{\prime}-s, y^{\prime}-y\right) \mathrm{d} s^{\prime} \mathrm{d} y^{\prime} \\
& \leq \sqrt{2} C_{T, k} \boldsymbol{p}_{t-s}(x-y)\left(\sum_{i=0}^{\infty} \gamma^{i} h_{i}(t-s)\right)^{1 / 2} .
\end{aligned}
$$

An application of Lemma 6.7 completes the proof of Theorem 6.4.

Remark 6.8. One can show that, for any fixed $(t, x) \in \mathbb{R}_{+} \times \mathbb{R}^{d}$, the mapping $(s, y) \mapsto$ $D_{s, y} u(t, x)$ from $(0, t) \times \mathbb{R}^{d}$ to $L^{k}(\Omega)$ is continuous for any $k \geq 2$. This follows from the fact that $D_{s, y} u(t, x)$ solves the following linear integral equation (see [11, Proposition 3.2] when $f$ is a nonnegative function):

$$
D_{s, y} u(t, x)=\boldsymbol{p}_{t-s}(x-y) \sigma(u(s, y))+\int_{(s, t) \times \mathbb{R}^{d}} \boldsymbol{p}_{t-r}(x-z) \sigma^{\prime}(u(r, z)) D_{s, y} u(r, z) \eta(\mathrm{d} r \mathrm{~d} z),
$$

obtained by applying the operator $D$ to the equation satisfied by $u(t, x)$. In this context, the asserted $L^{k}(\Omega)$-continuity is proved by resorting to usual arguments based on the Burkholder-Davis-Gundy inequality. We omit the many details. This continuity property is relevant though, for it allows us to appeal to Doob's theory of separability [22] in order to deduce the Lebesgue measurability of $(s, y) \mapsto D_{s, y} u(t, x)$.

\section{Proof of stationarity}

For every $\varphi \in C\left(\mathbb{R}_{+} \times \mathbb{R}^{d}\right)$ and $y \in \mathbb{R}^{d}$ define shift operators $\left\{\theta_{y}\right\}_{y \in \mathbb{R}^{d}}$ as follows:

$$
\left(\varphi \circ \theta_{y}\right)(t, x)=\varphi(t, x+y) .
$$

Clearly, $\theta:=\left\{\theta_{y}\right\}_{y \in \mathbb{R}^{d}}$ is a group under composition. The following result is used tacitly in the literature many times without explicit proof or even mention (see for example [17]). It also improves the assertion, observed by Dalang [19] that the 2-point correlation function of $x \mapsto u(t, x)$ is invariant under $\theta$. When $\sigma(z) \propto z$ the latter moment invariance (and more) can be deduced directly from an explicit Feynman-Kac type moment formula; see for example Chen, $\mathrm{Hu}$, and Nualart [9].

Lemma 7.1 (Spatial Stationarity). Suppose that $f$ either satisfies (1.4), or (1.8) for some $h \in \cup_{p>1} \mathcal{G}_{p}\left(\mathbb{R}^{d}\right)$, so that (1.1) has a unique random-field solution u; see Dalang [19] and Theorem 5.3. Then the random field $u \circ \theta_{y}$ has the same finite-dimensional distributions as $u$ for every $y \in \mathbb{R}^{d}$. In particular, for every $t \geq 0$, the finite-dimensional distributions of $\{u(t, x+y)\}_{x \in \mathbb{R}^{d}}$ do not depend on $y \in \mathbb{R}^{d}$.

Proof. The fact that (1.1) has a mild solution is another way to state that the transformation $\eta \mapsto u$ defines canonically a "solution map" $\mathbb{S}$ via $u=\mathbb{S}(\eta)$, where we recall $\eta$ denotes the driving noise. Recall also that the generalized Gaussian random field $\eta$ can be identified with a densely-defined isonormal Gaussian process $C_{c}\left(\mathbb{R}_{+} \times \mathbb{R}^{d}\right) \ni \varphi \mapsto \eta(\varphi)$ via Wiener integrals as follows:

$$
\eta(\varphi)=\int_{\mathbb{R}_{+} \times \mathbb{R}^{d}} \varphi \mathrm{d} \eta \quad \text { for all } \varphi \in \mathcal{H},
$$


where $\mathcal{H}$ is the Hilbert space introduced in $\S 6$. Since $C_{c}\left(\mathbb{R}_{+} \times \mathbb{R}^{d}\right) \ni \varphi \mapsto \eta(\varphi) \in L^{2}(\Omega)$ is a continuous linear mapping with respect to the $L^{2}\left(\mathbb{R}_{+} \times \mathbb{R}^{d}\right)$-norm, the preceding identifies $\eta$ completely provided only that we prescribe $\eta(\varphi)$ for every $\varphi \in C_{c}\left(\mathbb{R}_{+} \times \mathbb{R}^{d}\right)$. In this way, we can define a Gaussian noise $\eta_{y}$-one for every $y \in \mathbb{R}^{d}$-via

$$
\eta_{y}(\varphi)=\int_{\mathbb{R}_{+} \times \mathbb{R}^{d}} \varphi(t, x-y) \eta(\mathrm{d} t \mathrm{~d} x) \quad \text { for all } \varphi \in C_{c}\left(\mathbb{R}_{+} \times \mathbb{R}^{d}\right) .
$$

It is easy to check covariances in order to see that $\eta_{y}(\varphi)$ and $\eta(\varphi)$ have the same law; therefore, the noises $\eta$ and $\eta_{y}$ have the same law for every $y \in \mathbb{R}^{d}$. Also, it follows from the construction of the Walsh/Itô stochastic integral that for all $t \geq 0, x, y \in \mathbb{R}^{d}$, and Walsh-integrable random fields $\Psi$,

$$
\int_{(0, t) \times \mathbb{R}^{d}} \Psi(s, z-y) \eta(\mathrm{d} s \mathrm{~d} z)=\int_{(0, t) \times \mathbb{R}^{d}} \Psi(s, z) \eta_{y}(\mathrm{~d} s \mathrm{~d} z) \quad \text { a.s. }
$$

This can be proved by standard approximation arguments, using only the fact that (7.2) holds by (7.1) when $\Psi$ is a simple random field; see Walsh [53, Chapter 2].

Finally, we may combine (1.1) and (7.2) in order to see that for all $t \geq 0$ and $x, y \in \mathbb{R}^{d}$,

$$
\begin{aligned}
u(t, x+y) & =1+\int_{(0, t) \times \mathbb{R}^{d}} \boldsymbol{p}_{t-s}(x+y-z) \sigma(u(s, z-y+y)) \eta(\mathrm{d} s \mathrm{~d} z) \\
& =1+\int_{(0, t) \times \mathbb{R}^{d}} \boldsymbol{p}_{t-s}(x-z) \sigma(u(s, z+y)) \eta_{y}(\mathrm{~d} s \mathrm{~d} z) \quad \text { a.s. }
\end{aligned}
$$

This proves that $u \circ \theta_{y}=\mathbb{S}\left(\eta_{y}\right)$ a.s. for every $y \in \mathbb{R}^{d}$, where we recall $\mathbb{S}$ denotes the solution map in (1.1). Because $u$ is continuous, the preceding is another way to state the first assertion of the result. The second assertion follows from the first for elementary reasons.

Let us mention also the following simple fact.

Lemma 7.2. A stationary process $Y:=\{Y(x)\}_{x \in \mathbb{R}^{d}}$ is ergodic provided that

$$
\lim _{N \rightarrow \infty} \operatorname{Var}\left(f_{[0, N]^{d}} \prod_{j=1}^{k} g_{j}\left(Y\left(x+\zeta^{j}\right)\right) \mathrm{d} x\right)=0,
$$

for all integers $k \geq 1$, every $\zeta^{1}, \ldots, \zeta^{k} \in \mathbb{R}^{d}$, and all Lipschitz-continuous functions $g_{1}, \ldots, g_{k}: \mathbb{R} \rightarrow \mathbb{R}$ that satisfy (1.13).

Proof. Suppose $g_{1}, \ldots, g_{k}: \mathbb{R} \rightarrow \mathbb{R}$ are non-constant, Lipschitz-continuous functions, but do not necessarily satisfy (1.13). We first verify that (7.3) holds for these $g_{i}$ 's as well. Indeed, define

$$
\widetilde{g}_{j}(w):=\frac{g_{j}(w)-g_{j}(0)}{\operatorname{Lip}\left(g_{j}\right)} \quad \text { for all } j=1, \ldots, k \text { and } w \in \mathbb{R},
$$

and observe that $\widetilde{g}_{1}, \ldots, \widetilde{g}_{k}: \mathbb{R} \rightarrow \mathbb{R}$ satisfy (1.13), and hence (7.3) holds when we replace every $g_{i}$ with $\tilde{g}_{i}$. It is easy to see that

$$
\begin{aligned}
& f_{[0, N]^{d}} \prod_{j=1}^{k} g_{j}\left(Y\left(x+\zeta^{j}\right)\right) \mathrm{d} x \\
&=\sum_{E \subseteq\{1, \ldots, k\}} \prod_{l \in E} g_{l}(0) f_{[0, N]^{d}} \prod_{j \in\{1, \ldots, k\} \backslash E} \operatorname{Lip}\left(g_{j}\right) \widetilde{g}_{j}\left(Y\left(x+\zeta^{j}\right)\right) \mathrm{d} x,
\end{aligned}
$$


where a product over the empty set is defined as equal to one. For example, when $k=2$, we have

$$
\begin{aligned}
f_{[0, N]^{d}} g_{1}(Y(x & \left.\left.+\zeta^{1}\right)\right) g_{2}\left(Y\left(x+\zeta^{2}\right)\right) \mathrm{d} x \\
& =f_{[0, N]^{d}}\left[\operatorname{Lip}\left(g_{1}\right) \widetilde{g}_{1}\left(Y\left(x+\zeta^{1}\right)\right)+g_{1}(0)\right]\left[\operatorname{Lip}\left(g_{2}\right) \widetilde{g}_{2}\left(Y\left(x+\zeta^{2}\right)\right)+g_{2}(0)\right] \mathrm{d} x,
\end{aligned}
$$

which yields (7.4) upon expanding the product inside the integral.

Minkowski's inequality ensures that, for all random variables $X_{1}, \ldots, X_{M} \in L^{2}(\Omega)$,

$$
\operatorname{Var}\left(X_{1}+\cdots+X_{M}\right) \leq\left(\sum_{i=1}^{M} \sqrt{\operatorname{Var}\left(X_{i}\right)}\right)^{2} \leq M^{2} \max _{1 \leq i \leq M} \operatorname{Var}\left(X_{i}\right) .
$$

Thus, we see from (7.4) that

$$
\begin{aligned}
& \operatorname{Var}\left(f_{[0, N]^{d}} \prod_{j=1}^{k} g_{j}\left(Y\left(x+\zeta^{j}\right)\right) \mathrm{d} x\right) \\
& \leq 4^{k} \max _{E \subseteq\{1, \ldots, k\}} \prod_{l \in E} g_{l}^{2}(0) \cdot \operatorname{Var}\left(f_{[0, N]^{d}} \prod_{j \in\{1, \ldots, k\} \backslash E} \operatorname{Lip}\left(g_{j}\right) \widetilde{g}_{j}\left(Y\left(x+\zeta^{j}\right)\right) \mathrm{d} x\right) \\
& \rightarrow 0 \quad \text { as } N \rightarrow \infty,
\end{aligned}
$$

thanks to (7.3). This proves the assertion that if (7.3) holds when $g_{i}$ 's are Lipschitz and satisfy (1.13), then (7.3) continues to hold for non-constant, Lipschitz-continuous $g_{i}$ 's, even when they do not satisfy (1.13). And it is easy to see that "non-constant" can be removed from the latter assertion without changing its truth: We merely factor out of the variance the constant $g_{i}$ 's, and relabel the remaining $g_{j}$ 's, thus reducing the problem to the non-constant case.

We now apply the preceding with $g_{i}$ 's replaced with sines and cosines, in order to deduce from stationarity that

$$
\lim _{N \rightarrow \infty} f_{[0, N]^{d}} \exp \left\{i \sum_{j=1}^{k} z_{j} Y\left(x+\zeta^{j}\right)\right\} \mathrm{d} x=\mathrm{E}\left[\exp \left\{i \sum_{j=1}^{k} z_{j} Y\left(\zeta^{j}\right)\right\}\right] \quad \text { in } L^{2}(\Omega),
$$

for all $z_{1}, \ldots, z_{k} \in \mathbb{R}$ and $\zeta^{1}, \ldots, \zeta^{k} \in \mathbb{R}^{d}$. On the other hand, von Neumann's $L^{2}$ version of the ergodic theorem [51] tells us that

$$
\lim _{N \rightarrow \infty} f_{[0, N]^{d}} \exp \left\{i \sum_{j=1}^{k} z_{j} Y\left(x+\zeta^{j}\right)\right\} \mathrm{d} x=\mathrm{E}\left[\exp \left\{i \sum_{j=1}^{k} z_{j} Y\left(\zeta^{j}\right)\right\} \mid \mathcal{I}\right] \quad \text { in } L^{2}(\Omega),
$$

where $\mathcal{I}$ denotes the invariant $\sigma$-algebra of $Y$. Equate the preceding two displays, and apply the inversion theorem of Fourier transforms, in order to see that every random vector of the form $\left(Y\left(\zeta^{1}\right), \ldots, Y\left(\zeta^{k}\right)\right)$ is independent of $\mathcal{I}$. This implies that $\mathcal{I}$ is independent of the $\sigma$-algebra generated by $Y$, and in particular $\mathcal{I}$ is independent of itself. This in turn proves the result.

\section{Proofs of Theorem 1.1 (part 1), Theorems 1.3 and 1.6, and Corollary 1.5}

We are ready to begin the proof of the Poincaré-type inequalities of Theorems 1.6 and 1.7. Then we will show that, among other things, our Poincaré-type inequalities imply the desired spatial ergodicity of $u$. 
Proof of Theorems 1.6 and 1.7. We shall prove Theorems 1.6 and 1.7 at once, since one argument follows from the other after we make small adjustments.

Define

$$
V_{N}:=\operatorname{Var}\left(f_{[0, N]^{d}} \mathcal{G}(x) \mathrm{d} x\right), \quad \text { where } \mathcal{G}(x):=\prod_{j=1}^{k} g_{j}\left(u\left(t, x+\zeta^{j}\right)\right) \quad \text { for all } x \in \mathbb{R}^{d},
$$

so that $V_{N}=f_{[0, N]^{2 d}} \operatorname{Cov}(\mathcal{G}(x), \mathcal{G}(y)) \mathrm{d} x \mathrm{~d} y$. We plan to calculate $\operatorname{Cov}(\mathcal{G}(x), \mathcal{G}(y))$, pointwise, using the Clark-Ocone formula (Proposition 6.3). To this end, we apply the chain rule for the Malliavin derivative [46, Proposition 1.2.4] in order to see that

$$
D_{s, z} \mathcal{G}(x)=\sum_{j_{0}=1}^{k}\left(\prod_{\substack{j=1 \\ j \neq j_{0}}}^{k} g_{j}\left(u\left(t, x+\zeta^{j}\right)\right)\right) g_{j_{0}}^{\prime}\left(u\left(t, x+\zeta^{j_{0}}\right)\right) D_{s, z} u\left(t, x+\zeta^{j_{0}}\right) .
$$

The covariance structure of $\eta$ [see (1.2)] and Proposition 6.3 together ensure that, when $f$ is additionally a function,

$$
\begin{aligned}
|\operatorname{Cov}(\mathcal{G}(x), \mathcal{G}(y))| & =\left|\int_{0}^{t} \mathrm{~d} s \int_{\mathbb{R}^{d}} \mathrm{~d} z \int_{\mathbb{R}^{d}} \mathrm{~d} w \operatorname{E}\left\{\mathrm{E}\left(D_{s, z} \mathcal{G}(x) \mid \mathcal{F}_{s}\right) \cdot \mathrm{E}\left(D_{s, w} \mathcal{G}(y) \mid \mathcal{F}_{s}\right)\right\} f(z-w)\right| \\
& \leq \int_{0}^{t} \mathrm{~d} s \int_{\mathbb{R}^{d}} \mathrm{~d} z \int_{\mathbb{R}^{d}} \mathrm{~d} w\left\|D_{s, z} \mathcal{G}(x)\right\|_{2}\left\|D_{s, w} \mathcal{G}(y)\right\|_{2} \overline{\mathbf{f}}(z-w),
\end{aligned}
$$

whence

$$
V_{N} \leq f_{[0, N]^{2 d}} \mathrm{~d} x \mathrm{~d} y \int_{0}^{t} \mathrm{~d} s \int_{\mathbb{R}^{d}} \mathrm{~d} z \int_{\mathbb{R}^{d}} \mathrm{~d} w\left\|D_{s, z} \mathcal{G}(x)\right\|_{2}\left\|D_{s, w} \mathcal{G}(y)\right\|_{2} \overline{\mathbf{f}}(z-w),
$$

where $\overline{\mathbf{f}}$ is defined in the statement of Theorem 6.4, and is a function. If $f$ is a measure, then we can adapt the preceding. Since we have said this sort of thing before in this paper, without mentioning how to adapt, we make the adaptation to the measure case now by merely observing that, in general, the preceding covariance bound gets adapted to the following, and for the same reasons as above:

$$
V_{N} \leq f_{[0, N]^{2 d}} \mathrm{~d} x \mathrm{~d} y \int_{0}^{t}\langle\psi(s, x, \bullet), \psi(s, y, \bullet) * \overline{\mathbf{f}}\rangle_{L^{2}\left(\mathbb{R}^{d}\right)} \mathrm{d} s,
$$

where $\psi(s, x, z):=\left\|D_{s, z} \mathcal{G}(x)\right\|_{2}$. In any case, Theorems 5.3 and 6.4 together imply the existence of a real number $c=c(T, k)$ such that

$$
\begin{aligned}
\left\|D_{s, z} \mathcal{G}(x)\right\|_{2} & \leq \sum_{j_{0}=1}^{k}\left(\prod_{j=1, j \neq j_{0}}^{k}\left\|g_{j}\left(u\left(t, x+\zeta^{j}\right)\right)\right\|_{2 k}\right)\left\|D_{s, z} u\left(t, x+\zeta^{j_{0}}\right)\right\|_{2 k} \\
& \leq c \sum_{j=1}^{k} \boldsymbol{p}_{t-s}\left(x+\zeta^{j}-z\right),
\end{aligned}
$$

uniformly for all $0<s<t \leq T$ and $x, z \in \mathbb{R}^{d}$. Recall the probability density function $I_{N}$ from (3.15). The preceding can be now combined with Tonelli's theorem and the semigroup property of the heat kernel in order to yield

$$
\begin{aligned}
V_{N} & \leq c^{2} \sum_{j, \ell=1}^{k} f_{[0, N]^{2 d}} \mathrm{~d} x \mathrm{~d} y \int_{0}^{t} \mathrm{~d} s\left\langle\boldsymbol{p}_{t-s}\left(x+\zeta^{j}-\bullet\right), \boldsymbol{p}_{t-s}\left(y+\zeta^{\ell}-\bullet\right) * \overline{\mathbf{f}}\right\rangle_{L^{2}\left(\mathbb{R}^{d}\right)} \\
& =c^{2} \sum_{j, \ell=1}^{k} \int_{0}^{t}\left(\boldsymbol{p}_{2(t-s)} * I_{N} * \tilde{I}_{N} * \overline{\mathbf{f}}\right)\left(\zeta^{j}-\zeta^{\ell}\right) \mathrm{d} s .
\end{aligned}
$$


Since $I_{N} * \tilde{I}_{N} \in C_{c}\left(\mathbb{R}^{d}\right)$ and $I_{N} * \tilde{I}_{N}$ is nonnegative definite, the function $I_{N} * \tilde{I}_{N} * \overline{\mathbf{f}}$ is continuous and nonnegative-definite, whence also maximized at 0 . Because the $\mathrm{d} x$-integral of $\boldsymbol{p}_{2(t-s)}(x)$ is one, it follows that

$$
V_{N} \leq c^{2} k^{2} t\left(I_{N} * \tilde{I}_{N} * \overline{\mathbf{f}}\right)(0) \leq c^{2} k^{2} t \frac{\overline{\mathbf{f}}\left([-N, N]^{d}\right)}{N^{d}} ;
$$

see (3.17) for the last inequality. This completes the proof of (1.12) when $f$ satisfies Dalang's condition (1.4), as well as the proof of (1.14) when $f$ satisfies (1.8) for some $h \in \cup_{p>1} \mathcal{G}_{p}\left(\mathbb{R}^{d}\right)$.

We can now prove the remaining results from the Introduction.

Proof of part 1 of Theorem 1.1. Parts 2 and 3 of Theorem 1.1 were proved respectively in $\S 3.3$ and $\S 4$. We now conclude the proof of Theorem 1.1 by verifying its first part. With this in mind, suppose $f$ satisfies (1.4) and $\hat{f}(\{0\})=0$, equivalently,

$$
\lim _{N \rightarrow \infty} \frac{f\left([-N, N]^{d}\right)}{N^{d}}=0
$$

thanks to part 2 of Theorem 1.1, which has already been established. According to the above hypothesis and Theorem 1.6,

$$
\lim _{N \rightarrow \infty} \operatorname{Var}\left(f_{[0, N]^{d}} \prod_{j=1}^{k} g_{j}\left(u\left(t, x+\zeta^{j}\right)\right) \mathrm{d} x\right)=0,
$$

for all $t>0, \zeta^{1}, \ldots, \zeta^{k} \in \mathbb{R}^{d}$, and all Lipschitz functions $g_{1}, \ldots, g_{k}: \mathbb{R} \rightarrow \mathbb{R}$ that satisfy (1.13). Lemma 7.2 now implies that $u$ is spatially ergodic, and concludes the proof of part 1 of Theorem 1.1.

Proof of Theorem 1.3. As was the case also in the proof of Theorem 1.1, the asserted stationarity of the solution has been proved earlier in Lemma 7.1. Now suppose $f$ satisfies (1.8) for some $h \in \cup_{p>1} \mathcal{G}_{p}\left(\mathbb{R}^{d}\right)$. Proposition 3.4 tells us that $|h| *|\tilde{h}|$ is a function of positive type; thus, it vanishes at infinity. This immediately yields

$$
\lim _{N \rightarrow \infty} f_{[-N, N]^{d}}(|h| *|\tilde{h}|)(x) \mathrm{d} x=0,
$$

and hence (8.4) (see Theorem 1.7). An appeal to Lemma 7.2 ends the proof of Theorem 1.3.

Finally, we verify Corollary 1.5. The proof is elementary. We include it here however since the proof depends crucially on careful computation of the various exponents in (8.5)-(8.9) below.

Proof of Corollary 1.5. If $h \in L^{2}\left(\mathbb{R}^{d}\right)$ then we set $p=q=2$ to see that $h \in L_{\text {loc }}^{p}\left(\mathbb{R}^{d}\right)$ and

$$
\int_{0}^{1}\left(\|h\|_{L^{p}\left(\mathbb{B}_{r}\right)}\|h\|_{L^{q}\left(\mathbb{B}_{r}^{c}\right)}+\|h\|_{L^{2}\left(\mathbb{B}_{r}^{c}\right)}^{2}\right) \omega_{d}(r) \mathrm{d} r \leq 2\|h\|_{L^{2}\left(\mathbb{R}^{d}\right)}^{2} \int_{0}^{1} \omega_{d}(r) \mathrm{d} r,
$$

so that (1.10) holds thanks to the local integrability of $\omega_{d}$. Thus, it remains to consider the case when (1.11) holds. In that case, we appeal to (1.11) and integrate in spherical coordinates in order to see that

$$
\int_{\mathbb{B}_{r}}|h(x)|^{p} \mathrm{~d} x \lesssim \int_{0}^{r} s^{d-1-p(d+\alpha) / 2} \mathrm{~d} s \quad \text { for every } r \in(0,1) .
$$


Hence,

$$
h \in L_{\text {loc }}^{p}\left(\mathbb{R}^{d}\right) \text { iff } p<\frac{2 d}{d+\alpha} .
$$

Since $\alpha<d$, it follows that $2 d /(d+\alpha)>1$ and hence $h \in L_{\text {loc }}^{p}\left(\mathbb{R}^{d}\right)$ for every $p$ between 1 and $2 d /(d+\alpha)$. For every such $p$, (1.11) ensures that

$$
\|h\|_{L^{p}\left(\mathbb{B}_{r}\right)} \lesssim r^{(d / p)-(d+\alpha) / 2} \quad \text { for every } r \in(0,1) .
$$

Choose one such $p$ and define $q:=p /(p-1)$, so that $p^{-1}+q^{-1}=1$. Eq. (1.11) implies that, for every $r \in(0,1)$,

$$
\begin{aligned}
\int_{\mathbb{B}_{r}^{c}}|h(x)|^{q} \mathrm{~d} x & \leq \int_{r<\|x\|<1}|h(x)|^{q} \mathrm{~d} x+\int_{\|x\|>1}|h(x)|^{q} \mathrm{~d} x \\
& \lesssim \int_{r}^{1} t^{d-1-q(d+\alpha) / 2} \mathrm{~d} t+\int_{1}^{\infty} t^{d-1-q(d+\beta) / 2} \mathrm{~d} t,
\end{aligned}
$$

where the implied constants do not depend on $r \in(0,1)$. The first integral is convergent regardless of the choice of $p$ (hence also $q$ ). The second integral converges iff

$$
q>\frac{2 d}{d+\beta},
$$

which can certainly be arranged if $p$ were chosen sufficiently close to $1 .^{6}$ Choose and fix $p>1$ sufficiently close to 1 in order to ensure that (8.6) holds, whence

$$
\|h\|_{L^{q}\left(\mathbb{B}_{r}^{c}\right)} \lesssim r^{(d / q)-(d+\alpha) / 2} \quad \text { for every } r \in(0,1) .
$$

Finally, we may repeat the preceding with $q$ replaced everywhere with 2 in order to see that

$$
\|h\|_{L^{2}\left(\mathbb{B}_{r}^{c}\right)} \lesssim r^{-\alpha / 2} \quad \text { for every } r \in(0,1) .
$$

We may now combine (8.5), (8.7), and (8.8) in order to see that,

$$
\|h\|_{L^{p}\left(\mathbb{B}_{r}\right)}\|h\|_{L^{q}\left(\mathbb{B}_{r}^{c}\right)}+\|h\|_{L^{2}\left(\mathbb{B}_{r}^{c}\right)}^{2} \lesssim r^{-\alpha} \quad \text { for every } r \in(0,1) .
$$

Because $\alpha<2 \wedge d$, it follows that $h \in \mathcal{G}_{p}\left(\mathbb{R}^{d}\right)$ for all $p$ sufficiently close to one.

\section{Applications}

The Poincaré-type inequalities of Theorems 1.6 and 1.7 have many consequences other than those mentioned in Theorems 1.1 and 1.3. We conclude the paper by presenting two rather different applications of these Poincaré-type inequalities.

\subsection{Spatial mixing}

We say that $u$ is spatially mixing if the random field $u(t)$ is (weakly) mixing for every $t>0[23,42,51]$. Recall that this means that

$$
\lim _{\|x\| \rightarrow \infty} \operatorname{Cov}\left[\prod_{j=1}^{k} g_{j}\left(u\left(t, x+\zeta^{j}\right)\right), \prod_{l=1}^{k} g_{l}\left(u\left(t, \zeta^{l}\right)\right)\right]=0,
$$

for all integers $k \geq 1$, real numbers $t>0, \zeta^{1}, \ldots, \zeta^{k} \in \mathbb{R}^{d}$, and functions $g_{1}, \ldots, g_{k}$ of the form $g_{j}(w)=\mathbf{1}_{\left(-\infty, a_{j}\right]}(w)$ for $w \in \mathbb{R}$ and arbitrary $a_{1}, \ldots, a_{k} \in \mathbb{R}$. Our next result finds unimprovable conditions for spatial mixing of the solution to (1.1). When $d=1$ and $\sigma \equiv$ constant, our condition is sharp, and in agreement with classical results of Maruyama [42] on mixing properties of stationary Gaussian processes.

\footnotetext{
${ }^{6}$ To be concrete, we may select $1<p<d /(d-1)$ to ensure that $q>d$, so that (8.6) holds.
} 
Corollary 9.1. Suppose $f$ satisfies Dalang's condition (1.4). Then $u$ is spatially mixing if

$$
\lim _{\|x\| \rightarrow \infty}\left(\boldsymbol{v}_{\lambda} * f\right)(x)=0, \quad \text { equivalently if } \lim _{\|x\| \rightarrow \infty} \int_{\mathbb{R}^{d}} \frac{\mathrm{e}^{i x \cdot z}}{2 \lambda+\|z\|^{2}} \hat{f}(\mathrm{~d} z)=0,
$$

for some, hence all, $\lambda>0$. Moreover, (9.2) is a necessary and sufficient condition for the spatial mixing of $u$ in the case that $\sigma$ is a constant.

We pause and briefly examine condition (9.2) before we prove the corollary.

Example 9.2. If the spectral measure $\hat{f}$ is a function, then Dalang's condition (1.4) and the classical Riemann-Lebesgue lemma of Fourier analysis together guarantee that the second formulation in condition (9.2) holds. Thus, $u$ is spatially mixing whenever the underlying noise has a spectral density that satisfies Dalang's condition.

Example 9.3. If $f$ is a function that satisfies Dalang's condition (1.4) as well as parts 1 and 2 of Definition 3.1, then the proof of our next corollary can be easily adapted ${ }^{7}$ in order to prove that the first condition in (9.2) holds. In particular, $u$ is spatially mixing provided that the correlation $f$ is a function of positive type that satisfies Dalang's condition; and in fact condition 3 of Definition 3.1 is not needed for mixing to hold.

Proof of Corollary 9.1. We can approximate every $\mathbf{1}_{\left(-\infty, a_{j}\right]}$ in $L^{\infty}(\mathbb{R})$ from above and below by bounded Lipschitz-continuous functions in order to see that $u$ is spatially mixing if and only if (9.1) holds for all $k \geq 1$, real numbers $t>0, \zeta^{1}, \ldots, \zeta^{k} \in \mathbb{R}^{d}$, and Lipschitz-continuous functions $g_{1}, \ldots, g_{k}: \mathbb{R} \rightarrow \mathbb{R}$. In other words, it suffices to prove that

$$
\lim _{\|x\| \rightarrow \infty} \operatorname{Cov}(\mathcal{G}(x), \mathcal{G}(0))=0,
$$

where $\mathcal{G}$ is the random field was defined in (8.1), and where the functions $g_{1}, \ldots, g_{k}$ therein are Lipschitz continuous. We may, and will, assume further and without loss in generality that $g_{1}, \ldots, g_{k}$ satisfy (1.13). This can be justified using an argument that appeared earlier in the proof of Lemma 7.2.

We now use Theorem 1.6, in exactly the same manner that was used to derive (8.2), in order to find that for every $x \in \mathbb{R}^{d}$, and for $\psi(s, x, z):=\left\|D_{s, z} \mathcal{G}(x)\right\|_{2}$,

$$
\begin{aligned}
\operatorname{Cov}(\mathcal{G}(x), \mathcal{G}(0)) & \leq \int_{0}^{t}\langle\psi(s, x, \bullet), \psi(s, 0, \bullet) * f\rangle_{L^{2}\left(\mathbb{R}^{d}\right)} \mathrm{d} s \\
& \leq c^{2} \sum_{j, \ell=1}^{k} \int_{0}^{t}\left\langle\boldsymbol{p}_{t-s}\left(x+\zeta^{j}-\bullet\right), \boldsymbol{p}_{t-s}\left(\zeta^{\ell}-\bullet\right) * f\right\rangle_{L^{2}\left(\mathbb{R}^{d}\right)} \mathrm{d} s,
\end{aligned}
$$

for the same constant $c>0$ that appeared in (8.3). The semigroup property of the heat kernel now yields

$$
\begin{aligned}
\operatorname{Cov}(\mathcal{G}(x), \mathcal{G}(0)) & \leq c^{2} \sum_{j, \ell=1}^{k} \int_{0}^{t}\left(\boldsymbol{p}_{2 s} * f\right)\left(x+\zeta^{j}-\zeta^{\ell}\right) \mathrm{d} s \\
& \leq c^{2} \mathrm{e}^{2 \lambda t} \sum_{j, \ell=1}^{k} \int_{0}^{t} \mathrm{e}^{-2 \lambda s}\left(\boldsymbol{p}_{2 s} * f\right)\left(x+\zeta^{j}-\zeta^{\ell}\right) \mathrm{d} s \\
& \leq \frac{c^{2} \mathrm{e}^{2 \lambda t}}{2} \sum_{j, \ell=1}^{k}\left(\boldsymbol{v}_{\lambda} * f\right)\left(x+\zeta^{j}-\boldsymbol{\zeta}^{\ell}\right) .
\end{aligned}
$$

This demonstrates that the first condition in (9.2) ensures (9.3), and completes the proof of spatial mixing of $u$. Next, we verify that the two conditions in (9.2) are equivalent.

\footnotetext{
${ }^{7}$ Basically, one replaces the function $|h| *|\tilde{h}|$ everywhere in the proof of Corollary 9.4 by the function $f$.
} 
Because $\boldsymbol{p}_{s} \in \mathscr{S}\left(\mathbb{R}^{d}\right)$ for every $s>0$,

$$
\left(\boldsymbol{p}_{s} * f\right)(x)=\frac{1}{(2 \pi)^{d}} \int_{\mathbb{R}^{d}} \mathrm{e}^{i x \cdot z-s\|z\|^{2} / 2} \hat{f}(\mathrm{~d} z) \quad \text { for all } x \in \mathbb{R}^{d} .
$$

Multiply both sides by $\exp (-\lambda s)$ and integrate them over $s \in(0, \infty)$ to find that

$$
\left(\boldsymbol{v}_{\lambda} * f\right)(x) \propto \int_{\mathbb{R}^{d}} \frac{\mathrm{e}^{i x \cdot z}}{2 \lambda+\|z\|^{2}} \hat{f}(\mathrm{~d} z) \quad \text { for all } \lambda>0 \text { and } x \in \mathbb{R}^{d} .
$$

In order to complete the proof, suppose $\sigma \equiv c_{0}$ for some $c_{0}>0$, and assume that $u$ is spatially mixing. Then we can specialize (9.3) to deduce that for every $t>0$,

$$
\lim _{\|x\| \rightarrow \infty} \operatorname{Cov}[u(t, x), u(t, 0)]=0 .
$$

But (4.1) and (1.2) together imply that for every $x \in \mathbb{R}^{d}$ and $t, \lambda>0$,

$$
\begin{aligned}
\operatorname{Cov}[u(t, x), u(t, 0)] & =c_{0}^{2} \int_{0}^{t}\left\langle\boldsymbol{p}_{s}(x+\bullet), \boldsymbol{p}_{s} * f\right\rangle_{L^{2}\left(\mathbb{R}^{d}\right)} \mathrm{d} s \\
& =c_{0}^{2} \int_{0}^{t}\left(\boldsymbol{p}_{2 s} * f\right)(x) \mathrm{d} s \geq c_{0}^{2} \int_{0}^{t} \mathrm{e}^{-\lambda s}\left(\boldsymbol{p}_{2 s} * f\right)(x) \mathrm{d} s .
\end{aligned}
$$

On the one hand, the above and (9.4) together tell us that, for every $t>0$,

$$
\int_{0}^{t} \mathrm{e}^{-\lambda s}\left(\boldsymbol{p}_{2 s} * f\right)(x) \mathrm{d} s \rightarrow 0 \quad \text { as }\|x\| \rightarrow \infty .
$$

On the other hand,

$$
\begin{aligned}
\int_{t}^{\infty} \mathrm{e}^{-\lambda s}\left(\boldsymbol{p}_{2 s} * f\right)(x) \mathrm{d} s & =\frac{1}{(2 \pi)^{d}} \int_{t}^{\infty} \mathrm{e}^{-\lambda s} \mathrm{~d} s \int_{\mathbb{R}^{d}} \hat{f}(\mathrm{~d} z) \mathrm{e}^{i x \cdot z-s\|z\|^{2}} \\
& \propto \int_{\mathbb{R}^{d}} \frac{\mathrm{e}^{i x \cdot z-t\left(\lambda+\|z\|^{2}\right)}}{\lambda+\|z\|^{2}} \hat{f}(\mathrm{~d} z),
\end{aligned}
$$

which leads to the following crude bound, valid uniformly for all $t>0$ :

$$
\int_{t}^{\infty} \mathrm{e}^{-\lambda s}\left(\boldsymbol{p}_{2 s} * f\right)(x) \mathrm{d} s \lesssim \mathrm{e}^{-\lambda t} \int_{\mathbb{R}^{d}} \frac{\hat{f}(\mathrm{~d} z)}{\lambda+\|z\|^{2}} .
$$

Combine this bound with (9.5) in order to see that

$$
\limsup _{\|x\| \rightarrow \infty} \int_{0}^{\infty} \mathrm{e}^{-\lambda s}\left(\boldsymbol{p}_{2 s} * f\right)(x) \mathrm{d} s \lesssim \mathrm{e}^{-\lambda t} \int_{\mathbb{R}^{d}} \frac{\hat{f}(\mathrm{~d} z)}{\lambda+\|z\|^{2}} \quad \text { for every } t>0 .
$$

Let $t \rightarrow \infty$ and appeal to Dalang's condition (1.4) in order to see that the left-hand side is zero for every $\lambda>0$. This concludes the proof.

Corollary 9.4. If $f$ satisfies (1.8) for some $h \in \cup_{p>1} \mathcal{G}_{p}\left(\mathbb{R}^{d}\right)$, then $u$ is spatially mixing.

Proof. Notice that by applying Theorem 1.7 instead of Theorem 1.6 (as what we have done in the transition from the proof of Theorem 1.1 to that of Theorem 1.3), Corollary 9.4 follows once one can establish that

$$
\lim _{\|x\| \rightarrow \infty} \int_{\mathbb{R}^{d}} \boldsymbol{v}_{1}(y)(|h| *|\tilde{h}|)(x-y) \mathrm{d} y=0 .
$$


On the one hand, since $|h| *|\tilde{h}|$ vanishes uniformly at infinity [Proposition 3.4] and $\boldsymbol{v}_{1}$ is a probability density function,

$$
\int_{\|y\|<\|x\| / 2} \boldsymbol{v}_{1}(y)(|h| *|\tilde{h}|)(x-y) \mathrm{d} y \leq \sup _{\|w\|>\|x\| / 2}(|h| *|\tilde{h}|)(w)=o(1) \quad \text { as }\|x\| \rightarrow \infty .
$$

On the other hand, a similar argument shows that

$$
\begin{aligned}
& \int_{\|y\|>\|x\| / 2} \boldsymbol{v}_{1}(y)(|h| *|\tilde{h}|)(x-y) \mathrm{d} y \\
& =\int_{\substack{\|y\|>\|x\| / 2 \\
\|y-x\|<\|x\| / 2}} \boldsymbol{v}_{1}(y)(|h| *|\tilde{h}|)(x-y) \mathrm{d} y+\int_{\substack{\|y\|>\|x\| / 2 \\
\|y-x\|>\|x\| / 2}} \boldsymbol{v}_{1}(y)(|h| *|\tilde{h}|)(x-y) \mathrm{d} y \\
& =\int_{\substack{\|y\|>\|x\| / 2 \\
\|y-x\|<\|x\| / 2}} \boldsymbol{v}_{1}(y)(|h| *|\tilde{h}|)(x-y) \mathrm{d} y+o(1) \quad \text { as }\|x\| \rightarrow \infty .
\end{aligned}
$$

Let $\Xi(x)$ denote the final integral in the above. It remains to prove that $\Xi(x) \rightarrow 0$ as $\|x\| \rightarrow \infty$. Since $\boldsymbol{v}_{1}(y)$ decreases monotonically as $\|y\|$ increases,

$$
\Xi(x) \leq \mathcal{V}(\|x\| / 2) \int_{\|y\|<\|x\| / 2}(|h| *|\tilde{h}|)(y) \mathrm{d} y,
$$

where

$$
\mathcal{V}(a)=\frac{1}{(2 \pi)^{d / 2}} \int_{0}^{\infty} s^{-d / 2} \exp \left(-s-\frac{a^{2}}{2 s}\right) \mathrm{d} s \propto a^{-(d-2) / 2} K_{(d-2) / 2}(a \sqrt{2}), \quad a>0,
$$

and $K_{\nu}$ denotes the modified Bessel function of the second kind. Elementary asymptotic evaluations imply that $K_{(d-2) / 2}(a) \lesssim a^{-1 / 2} \exp (-a)$ for all $a>1$ (see [47, 10.25.3]), whence $\mathcal{V}(a) \lesssim a^{-(d-1) / 2} \exp (-a \sqrt{2})$. Consequently,

$$
\Xi(x) \lesssim \mathrm{e}^{-\|x\| \sqrt{2}} \int_{\|y\|<\|x\| / 2}(|h| *|\tilde{h}|)(y) \mathrm{d} y,
$$

uniformly for all $x \in \mathbb{B}_{2}^{c}$ (with room to spare). Because $c:=\sup _{y \in \mathbb{B}_{1}^{c}}(|h| *|\tilde{h}|)(y)$ is finite (see Proposition 3.4),

$$
\int_{\|y\|<\|x\| / 2}(|h| *|\tilde{h}|)(y) \mathrm{d} y \leq \int_{\mathbb{B}_{1}}(|h| *|\tilde{h}|)(y) \mathrm{d} y+c \int_{1<\|y\|<\|x\| / 2} \mathrm{~d} y \lesssim\|x\|^{d},
$$

as $\|x\| \rightarrow \infty$. This proves that $\Xi(x) \lesssim\|x\|^{d} \exp (-\|x\| \sqrt{2})=o(1)$, as $\|x\| \rightarrow \infty$, which completes the proof.

\subsection{Intermittency}

In this final section we include an additional application of our Poincaré inequalities. In order to simplify the exposition, we consider (1.1) in the case of the parabolic Anderson model driven by space-time white noise. That is, we propose to study the SPDE,

$$
\partial_{t} u=\frac{1}{2} \partial_{x}^{2} u+u \eta \quad \text { on }(0, \infty) \times \mathbb{R},
$$

subject to $u(0) \equiv 1$, where

$$
\mathrm{E}[\eta(t, x) \eta(s, y)]=\delta_{0}(t-s) \delta_{0}(x-y) \quad \text { for every } s, t>0 \text { and } x, y \in \mathbb{R} .
$$

It is well known that $u(t, x)>0$ for all $t>0$ and $x \in \mathbb{R}$ off a single P-null set (see Mueller $[44,45])$, and that the solution is unbounded at all times $t>0$, viz.,

$$
\lim _{N \rightarrow \infty} \sup _{x \in[0, N]} u(t, x)=\infty \quad \text { a.s. for every } t>0 .
$$


In fact, Chen [12, Theorem 1.7] has established the following improvement of (9.8):

$$
\lim _{N \rightarrow \infty} \sup _{x \in[0, N]} \frac{\log u(t, x)}{(\log N)^{2 / 3}}=\frac{3}{4}\left(\frac{2 t}{3}\right)^{2 / 3} \quad \text { a.s. }{ }^{8}
$$

Conus et al [15] have studied the Lebesgue measure of the set of $x \in[0, N]$ where $u(t, x)$ is almost as tall as the maximum possible, as given in (9.9). The following verifies one of their conjectures; see [15, see (1.5)].

Corollary 9.5. Choose and fix some $t>0$, and define $d(\alpha):=4 \alpha 3^{-3 / 2} \sqrt{6 / t}$ for all $\alpha>0$. Whenever $d(\alpha)<1 / 2$, the following holds almost surely:

$$
\lim _{N \rightarrow \infty} \frac{1}{\log N} \log \left(\int_{0}^{N} \mathbf{1}_{\left\{u(t, x)>\exp \left[(\alpha \log N)^{2 / 3}\right]\right\}} \mathrm{d} x\right)=1-d(\alpha) .
$$

The quantity on the left-hand side of (9.10) is a kind of "macroscopic fractal dimension" for the set $\mathscr{P}(\theta)$ of $x \in \mathbb{R}$ such that $u(t, x)$ exceeds $\exp \left\{\theta(\log |x|)^{2 / 3}\right\}$. In this way we can see that the "fractal dimension formula" (9.10) yields a "codimension formula" for the macroscopic Hausdorff dimension of $\mathscr{P}(\theta)$; this should be compared to the related dimension formulas of Khoshnevisan, Kim, and Xiao [39, Theorem 1.2].

Proof. Before we begin, let us observe that Theorem 1.6 implies that ${ }^{9}$

$$
\sup _{g: \operatorname{Lip}(g) \leq 1} \sup _{N>0} \operatorname{Var}\left(\frac{1}{\sqrt{N}} \int_{0}^{N} g(u(t, x)) \mathrm{d} x\right)<\infty .
$$

Now we choose and fix $\alpha>0$, and define $a_{N}:=\exp \left\{\left(\alpha \log _{+} N\right)^{2 / 3}\right\}$ for every $N>0$. We plan to apply (9.11) with $g$ replaced by either $g_{N}$ or $G_{N}$, where

$$
G_{N}(z):=1 \wedge\left(z-a_{N}+1\right)_{+} \text {and } g_{N}(z):=1 \wedge\left(z-a_{N}\right)_{+} .
$$

According to Theorem 5.5 of Chen [12], $a^{-3 / 2} \log \mathrm{P}\left\{u(t, 0)>\mathrm{e}^{a}\right\} \rightarrow-d(1)$ as $a \rightarrow \infty$. Because

$$
g_{N} \leq \mathbf{1}_{\left[a_{N}, \infty\right)} \leq G_{N} \quad \text { for every } N>0,
$$

it immediately follows from stationarity that, as $N \rightarrow \infty$,

$$
\mathrm{E} \int_{0}^{N} g_{N}(u(t, x)) \mathrm{d} x=N^{1-d(\alpha)+o(1)} \quad \text { and } \quad \mathrm{E} \int_{0}^{N} G_{N}(u(t, x)) \mathrm{d} x=N^{1-d(\alpha)+o(1)} .
$$

On the other hand, both $g_{N}$ and $G_{N}$ are 1-Lipschitz. Therefore, (9.11) holds when $g=g_{N}$ as well as $g=G_{N}$. Because of this, Chebyshev's inequality ensures that for every fixed $\epsilon \in(0,1)$,

$\mathrm{P}\left\{\left|\int_{0}^{N} g_{N}(u(t, x)) \mathrm{d} x-\mathrm{E} \int_{0}^{N} g_{N}(u(t, x)) \mathrm{d} x\right|>\epsilon \mathrm{E} \int_{0}^{N} g_{N}(u(t, x)) \mathrm{d} x\right\} \leq N^{-1+2 d(\alpha)+o(1)}$,

as $N \rightarrow \infty$. And the same estimate is valid when we replace $g_{N}$ everywhere by $G_{N}$. These facts and (9.12) together show that, if $2 d(\alpha)<1$, then (9.10) holds in probability. A standard subsequencing and blocking argument can be used to prove a.s.-convergence in (9.10). We skip this part, although we caution that some care is required in order to carry this out properly. This concludes the proof.

\footnotetext{
${ }^{8}$ Chen [12] proves this fact with $\sup _{x \in[-N, N]} u(t, x)$ in place of $\sup _{x \in[0, N]} u(t, x)$. The present statement is proved in the same way, however.

${ }^{9}$ Theorem 1.6 requires also that $g(0)=0$. We obtain (9.11) by replacing $g$ by $g-g(0)$, without altering the value of the variance.
} 
Spatial ergodicity for SPDEs

\section{References}

[1] Bertini, L. and Cancrini, N.: The stochastic heat equation: Feynman-Kac formula and intermittence. J. Statist. Phys. 78(5-6), (1995), 1377-1401. MR1316109

[2] Burkholder, D. L.: Martingale transforms. Ann. Math. Statist. 37, (1966), 1494-1504. MR0208647

[3] Burkholder, D. L., Davis, B. J., and Gundy, R. F.: Integral inequalities for convex functions of operators on martingales. In: Proceedings of the Sixth Berkeley Symposium on Mathematical Statistics and Probability II, 223-240, University of California Press, Berkeley, California, 1972. MR0400380

[4] Burkholder, D. L. and Gundy, R. F.: Extrapolation and interpolation of quasi-linear operators on martingales. Acta Math. 124, (1970), 249-304. MR0440695

[5] Capitaine, M., Hsu, E. P. and Ledoux, M.: Martingale representation and a simple proof of the logarithmic Sobolev inequalities on path space. Electr. Comm. Probab. 2, (1997), 71-81. MR1484557

[6] Cardon-Weber, C. and Millet, A.: On strongly Petrovskiı's parabolic SPDEs in arbitrary dimension and application to the stochastic Cahn-Hilliard equation. J. Theoret. Probab. 17(1), (2004), 1-49. MR2054575

[7] Carlen, E. and Krée, P.: $L^{p}$ estimates on iterated stochastic integrals. Ann. Probab. 19(1), (1991), 354-368. MR1085341

[8] Carmona, R. A. and Molchanov, S. A.: Parabolic Anderson Problem and Intermittency. Memoires of the Amer. Math. Soc. 108, American Mathematical Society, Rhode Island, 1994. MR1185878

[9] Chen, L., Hu, Y. and Nualart, D.: Two-point correlation function and Feynman-Kac formula for the stochastic heat equation. Potential Anal. 46(4), (2017), 779-797. MR3636598

[10] Chen, L. and Huang, J.: Comparison principle for stochastic heat equation on $\mathbb{R}^{d}$. Ann. Probab. 47(2), (2019), 989-1035. MR3916940

[11] Chen, L. and Huang, J.: Regularity and strict positivity of densities for the stochastic heat equation on $\mathbb{R}^{d}$. arXiv:1902.02382.

[12] Chen, X.: Spatial asymptotics for the parabolic Anderson models with generalized time-space Gaussian noise. Ann. Probab. 44(2), (2016), 1535-1598. MR3474477

[13] Chen, X., Hu, Y., and Nualart, D.: Spatial asymptotics for the parabolic Anderson model driven by a Gaussian rough noise. Electron. J. Probab. 22(65), (2017), 38 pp. MR3690290

[14] Ocone, D.: Malliavin calculus and stochastic integral representation of diffusion processes. Stochastics 12, (1984), 161-185. MR0749372

[15] Conus, D., Joseph, M., and Khoshnevisan, D.: Correlation-length bounds, and estimates for intermittent islands in parabolic SPDEs. Electr. J. Probab. 17(102), (2012), 1-15. MR3005720

[16] Conus, D. and Khoshnevisan, D.: On the existence and position of the farthest peaks of a family of stochastic heat and wave equations. Probab. Theory Rel. Fields 152(3-4), (2012), 681-701. MR2892959

[17] Conus, D., Joseph, M., and Khoshnevisan, D.: On the chaotic character of the stochastic heat equation, before the onset of intermitttency. Ann. Probab. 41(3B), (2013), 2225-2260. MR3098071

[18] Conus, D., Joseph, M., Khoshnevisan, D., and Shiu, S.: On the chaotic character of the stochastic heat equation, II. Probab. Theory Related Fields 156(3-4), (2013), 483-533. MR3078278

[19] Dalang, R. C.: Extending the martingale measure stochastic integral with applications to spatially homogeneous s.p.d.e.'s. Electron. J. Probab. 4(6), (1999), 29 pp. MR1684157

[20] Dalang, R. C. and Frangos, N. E.: The stochastic wave equation in two spatial dimensions. Ann. Probab. 26(1), (1998), 187-212. MR1617046

[21] Davis, B.: On the $L^{p}$ norms of stochastic integrals and other martingales. Duke Math. J. 43(4), (1976), 697-704. MR0418219 
Spatial ergodicity for SPDEs

[22] Doob, J. L.: Stochastic Processes. Reprint of the 1953 original. John Wiley \& Sons, Inc., New York, 1990, viii+654. MR1038526

[23] Dym, H. and McKean, H. P.: Gaussian Processes, Function Theory, and the Inverse Spectral Problem. Probability and Mathematical Statistics, Vol. 31. Academic Press, New York-London, 1976. xi+335 pp. MR0448523

[24] Edgar, G. A., and Sucheston, L.: Stopping Times and Directed Processes. Cambridge University Press, Cambridge, 1992. MR1191395

[25] Foondun, M. and Khoshnevisan, D.: Intermittence and nonlinear parabolic stochastic partial differential equations. Electron. J. Probab. 14(21), (2009), 548-568. MR2480553

[26] Foondun, M. and Khoshnevisan, D.: On the stochastic heat equation with spatially-colored random forcing. Trans. Amer. Math. Soc. 365(1), (2013), 409-458. MR2984063

[27] Gaveau, B. and Trauber, P.: L'intégrale stochastique comme opérateur de divergence dans l'espace fonctionnel. J. Functional Anal. 46, (1982), 230-238. MR0660187

[28] Hawkes, J.: Potential theory of Lévy processes. Proc. London Math. Soc. (3) 38, no. 2, (1979), 335-352. MR0531166

[29] Hawkes, J.: Some geometric aspects of potential theory. In: Stochastic Analysis and Applications (Swansea, 1983), 130-154, Lecture Notes in Math. 1095 Springer, Berlin, 1984. MR0777518

[30] Hu, Y., Huang, J., Lê, K., Nualart, D., and Tindel, S.: Stochastic heat equation with rough dependence in space. Ann. Probab. 45(6B), (2017), 4561-4616. MR3737918

[31] Hu, Y., Huang, J., Nualart, D., and Tindel, S.: Stochastic heat equations with general multiplicative Gaussian noises: Hölder continuity and intermittency. Electron. J. Probab. 20(55), (2015), 50 pp. MR3354615

[32] Huang, J., Lê, K., and Nualart, D.: Large time asymptotics for the parabolic Anderson model driven by space and time correlated noise. Stoch. Partial Differ. Equ. Anal. Comput. 5(4), (2017), 614-651. MR3736656

[33] Huang, J., Nualart, D., and Viitasaari, L.: A central limit theorem for the stochastic heat equation. Stochastic Process. Appl. 131, (2020), 7170-7184. MR4167203

[34] Huang, J., Nualart, D., Viitasaari, L. and Zheng, G.: Gaussian fluctuations for the stochastic heat equation with colored noise. Stoch. Partial Differ. Equ. Anal. Comput. 8, (2020), 402-421. MR4098872

[35] Kahane, J.P.: Some Random Series of Functions. Second edition. Cambridge University Press, Cambridge, 1985. MR0833073

[36] Karczewska, A. and Zabczyk, J.: A note on stochastic wave equations. In: Evolution Equations and Their Applications in Physical and Life Sciences (Bad Herrenalb, 1998), 501-511, Lecture Notes in Pure and Appl. Math. 215 Dekker, NY, 2001. MR1818028

[37] Khoshnevisan, D.: Analysis of Stochastic Partial Differential Equations. CBMS Regional Conference Series in Mathematics, 119. American Mathematical Society, Providence, RI, 2014. viii+116. MR3222416

[38] Khoshnevisan, D.: Multiparameter Processes. Springer Monographs in Mathematics. Springer-Verlag, New York, 2002. xx+584 pp. MR1914748

[39] Kim, K., Khoshnevisan, D., and Xiao, Y.: Intermittency and multifractality: A case study via stochastic PDEs. Ann. Probab. 45(6A), (2017), 3697-3751. MR3729613

[40] Kim, K., Khoshnevisan,D., and Xiao, Y.: A macroscopic multifractal analysis of parabolic stochastic PDEs. Comm. Math. Phys. 360, (2018), 307-346. MR3795193

[41] Lépingle, D. and Ouvrard, J. Y.: Martingales browniennes hilbertiennes. C. R. Acad. Sci. Paris Sér. A-B 276, (1973), A1225-A1228. MR0317406

[42] Maruyama, G.: The harmonic analysis of stationary stochastic processes. Mem. Faculty Sci. Kyushu Univ. Ser. A. 4, (1949), 45-106. MR0032127

[43] Millet, A. and Sanz-Solé, M.: A stochastic wave equation in two space dimension: smoothness of the law. Ann. Probab. 27(2), (1999), 803-844. MR1698971

[44] Mueller, C.: On the support of solutions to the heat equation with noise. Stochastics Stochastics Rep. 37(4), (1991), 225-245. MR1149348 
[45] Mueller, C.: Some tools and results for parabolic stochastic partial differential Equations. In: A Minicourse on Stochastic Partial Differential Equations, pp. 111-144, Lecture Notes in Math. 1962 Springer, Berlin, 2009. MR2508775

[46] Nualart, D.: The Malliavin Calculus and Related Topics. Second edition. Probability and its Applications (New York). Springer-Verlag, Berlin, 2006. MR2200233

[47] Olver, F. W. J., Lozier, D. W., Boisvert, R. F. and Clark, C. W.: NIST Handbook of Mathematical Functions. Cambridge Univ. Press, Cambridge, UK, 2010. MR2723248

[48] Ouvrard, J. Y.: Représentation de martingales vectorielles de carré intégrable à valeurs dans des espaces de Hilbert réels séparables. Z. Wahrscheinlichkeitstheorie und Verw. Gebiete 33(3), (1975/76), 195-208. MR0394862

[49] Peszat, S.: The Cauchy problem for a nonlinear stochastic wave equation in any dimension. $J$. Evol. Equ. 2(3), (2002), 383-394. MR1930613

[50] Peszat, S. and Zabczyk, J.: Nonlinear stochastic wave and heat equations. Probab. Theory Related Fields 116(3), (2000), 421-443. MR1749283

[51] Peterson, K.: Ergodic Theory. Cambridge University Press, Cambridge, 1983. MR0833286

[52] Stein, E. M.: Singular Integrals and Differentiability Properties of Functions. Princeton University Press, Princeton, N.J, 1970. MR0290095

[53] Walsh, J. B.: An Introduction to Stochastic Partial Differential Equations. Ècole d'été de probabilités de Saint-Flour, XIV-1984, 265-439, In: Lecture Notes in Math. 1180, Springer, Berlin, 1986. MR0876085

[54] Zeldovich, Ya. B., Molchanov, S. A., Ruzmaikin, A. A. and Sokolov, D. D.: Intermittency, diffusion, and generation in a nonstationary random medium, Sov. Sci. Rev. C. Math. Phys. 7, (1988), 1-110. MR1128327

[55] Zeldovich, Ya. B., Molchanov, S. A., Ruzmaikin, A. A., and Sokolov, D. D.: Intermittency of passive fields in random media, J. of Experimental and Theoretical Physics [actual journal title: Журнал експериментальнойи теоретическойфизики] 89[6(12)], (1985), 2061-2072. (In Russian)

[56] Zeldovich, Ya. B., Ruzmaikin, A. A., and Sokoloff, D. D.: The Almighty Chance, World Scientific Lecture Notes in Physics, vol. 20, Singapore, 1990. MR1141627

Acknowledgments. F. Pu is grateful to University of Utah where the work was carried out. 


\section{Electronic Journal of Probability Electronic Communications in Probability}

\section{Advantages of publishing in EJP-ECP}

- Very high standards

- Free for authors, free for readers

- Quick publication (no backlog)

- Secure publication $\left(\mathrm{LOCKSS}^{1}\right)$

- Easy interface (EJMS²)

\section{Economical model of EJP-ECP}

- Non profit, sponsored by $\mathrm{IMS}^{3}, \mathrm{BS}^{4}$, ProjectEuclid ${ }^{5}$

- Purely electronic

\section{Help keep the journal free and vigorous}

- Donate to the IMS open access fund ${ }^{6}$ (click here to donate!)

- Submit your best articles to EJP-ECP

- Choose EJP-ECP over for-profit journals

\footnotetext{
${ }^{1}$ LOCKSS: Lots of Copies Keep Stuff Safe http://www. lockss.org/

${ }^{2}$ EJMS: Electronic Journal Management System http://www.vtex.lt/en/ejms.html

${ }^{3}$ IMS: Institute of Mathematical Statistics http://www.imstat.org/

${ }^{4}$ BS: Bernoulli Society http://www. bernoulli-society.org/

${ }^{5}$ Project Euclid: https://projecteuclid.org/

${ }^{6}$ IMS Open Access Fund: http://www.imstat.org/publications/open.htm
} 